\title{
A stochastic scaled boundary finite element method
}

\author{
X.Y. Long ${ }^{\mathrm{a}}$, C. Jiang ${ }^{\mathrm{a} *}$, X. Han ${ }^{\mathrm{a}}$, W. Gao ${ }^{\mathrm{b}}$, and J. Liu ${ }^{\mathrm{a}}$ \\ ${ }^{a}$ State Key Laboratory of Advanced Design and Manufacturing for Vehicle Body, College of \\ Mechanical and Vehicle Engineering, Hunan University, Changsha City, P. R. China 410082 \\ ${ }^{\mathrm{b}}$ School of Civil and Environmental Engineering, The University of New South Wales, \\ Sydney, NSW 2052, Australia
}

\begin{abstract}
By extending the existing scaled boundary finite element method (SBFEM) into the random field problem, a stochastic scaled boundary finite element method (SSBFEM) is developed in this paper to predict the structural responses with randomly distributed material properties. The analyzed domain is discretized into a number of polygons modeled as sub-domains of the SBFEM so that the non-homogenous material properties are possible to be considered in establishment of the constitutive equation. The random field represented by the Karhunen-Loève (KL) expansion is then directly incorporated into both the equilibrium equation of the SBFEM and its sensitivity analysis formulation. The perturbation method is employed to calculate the statistical moments of the structural responses and a semi-analytical sensitivity analysis method is used to efficiently calculate the gradients involved. Particularly, the proposed method is successfully applied to the stochastic fracture analysis with spatially varying random material properties. Four numerical examples are investigated to demonstrate the validity of the proposed method.
\end{abstract}

Keywords: Stochastic scaled boundary finite element method; Random field; Perturbation method; Sensitivity analysis; Stochastic fracture mechanics

\section{Introduction}

The SBFEM developed by Song and Wolf $[1,2]$ is a semi-analytical method which is powerful for structural analysis with stress singularity or unbounded foundation media. For elasticity problems

\footnotetext{
* Corresponding author.
}

E-mail address: jiangc@hnu.edu.cn (C. Jiang), hanxu@hnu.edu.cn (X. Han).

Tel: +86073188823325 
with simple geometries where the scaling center can be visible from the whole boundary, it discretizes the domain boundaries only, as in the boundary element method (BEM), but does not need fundamental solutions, as in the finite element method (FEM). For more complex problems, the interior of the domain can be discretised into subdomains so as to satisfy the scaling requirement and other requirements. In the last two decades, the SBFEM has achieved great success. Wolf [3] analyzed the response of unbounded soil using SBFEM. Birk and Behnke [4] extended the SBFEM for the analysis of 3D-layered continua. Bazyar and Talebi [5] applied SBFEM to the analysis of unsteady seepage flow problems. Tao et al. [6] solved the boundary-value problem composed of short-crested waves diffracted by a vertical circular cylinder by SBFEM. He et al. [7] developed an element-free Galerkin scaled boundary method for solving steady-state heat transfer problems. Natarajan et al. [8] combined the concept of isogeometric analysis and SBFEM. Concerning the analytical representation of the stress singularities in SBFEM, Song and Wolf [9] investigated the singular stress occurring at cracks in anisotropic multi-materials. Yang [10], Ooi and Yang [11, 12], and Dai et al. [13] used the SBFEM to analyze crack propagation problems. Ooi et al. [14] developed a novel polygon-based SBFEM formulation for elasto-plastic problems.

In the above-mentioned works, generally all the involved parameters such as the loads, material properties, and geometrical characteristics, were given definite values so that the structural responses are deterministic ones. However, due to the manufacturing errors and unpredictable environmental factors, the modelings of many engineering problems need the consideration of inherent uncertain parameters as inputs. To address this problem, in the recent years the SBFEM has been successfully applied to the stochastic response analysis, especially to the probabilistic fracture mechanics analysis for its advantages that only the boundaries require to be discretized and a large perturbation of the crack tip location can be modeled without re-meshing. For examples, Chowdhury et al. $[15,16]$ studied the probabilistic failure of the cracked structures based on SBFEM, in which the uncertainties of the loads, material properties and geometric characteristics were considered. Jiang et al. [17] developed a probability-interval hybrid reliability analysis method for cracked structures by SBFEM. Long et al. [18] proposed an efficient stochastic response analysis method using SBFEM and applied it to the probabilistic fracture mechanics. Long et al. [19] employed the Monte Carlo simulation (MCS) in combination with SBFEM to analyze the 
stochastic fracture problem.

Indeed, the current studies [15-19] have promoted the development of the SBFEM in the field of uncertain analysis to a certain extent. Nevertheless, most of the studies are focused on the stochastic structural analysis by modeling all the uncertainties as random variables, while less work is conducted for the random field analysis using SBFEM, in which the input uncertain parameters are varied randomly across the space. In practice, a wide class of materials (soils, rocks, ceramics, composites, etc.) exhibit spatial randomness of their properties. For example, the material properties of the reinforced concrete (RC) are varied across the space due to the random distributions of the RC members (steel fiber, concrete fiber, etc.) [20]. The local stiffness of the ceramics is spatially fluctuated due to the presence of initial porosity induced by sintering [21]. The material properties of the composite materials, especially the fiber reinforced composites, normally show considerable display inherent scatter owing to the uncontrollable factors in the manufacturing process [22]. It is, therefore, very important to develop a method to calculate the global statistical characteristics of the structural responses by considering the spatial variability of the material properties. In the last several decades, the stochastic finite element method (SFEM) in which the uncertainties are quantified by random fields has received considerable attention [23-29]. A comprehensive overview of the SFEM can be referred to Refs. [27] and [29]. Though great achievements have been made about the SFEM, most of the existing studies focus on the generation of realistic random fields. To the best knowledge of the authors, few work has been performed on the fracture problems under random fields since the mesh of the FEM around the crack tip generally requires special treatment which may lead to complex discretizations. On the other hand, for the SBFEM, no special mesh treatment around the crack tip is needed and highly accurate stress intensity factors (SIFs) can be calculated directly by definition with no singular integrals. This makes it possible to efficiently deal with the stochastic fracture problems with randomly distributed material properties by combining SBFEM and random field theory. Hence, it is appealing to develop a new method based on SBFEM in order to efficiently evaluate the global stochastic structural responses under random fields, which may play an important role in the fields of the stochastic structural response analysis and, in particular, the stochastic fracture mechanics analysis.

This paper aims to develop a stochastic scaled boundary finite element method (SSBFEM) to 
efficiently calculate the statistical characteristics of the structural responses with spatially varying material properties. In addition, the proposed SSBFEM is applied to deal with the stochastic fracture problems. The remainder of this paper is organized as follows. Section 2 summarizes the basic theory of the existing SBFEM; Section 3 presents the formulation of the proposed SSBFEM; Sections 4 shows the application of the SSBFEM to stochastic fracture problems; Section 5 provides the analysis of four numerical examples.

\section{The scaled boundary finite element method (SBFEM)}

The basic concept of the SBFEM [30-32] is necessary to be first introduced. Figure 1(a) shows a domain of arbitrary geometry modeled by a few sub-domains. Each sub-domain has a scaling center from which its whole boundary is visible. As shown in Fig. 1(b), the SBFEM introduces a coordinate system $(\xi, \eta)$ by scaling the domain boundary relative to the scaling center O: the radial coordinate $\xi$ points to the boundary from the scaling center where its value is zero while its value is 1 on the boundary, and the circumferential coordinate $\eta$ is along the boundary direction. The displacement field in a sub-domain is approximated analytically in the radial direction and in the FEM sense in the circumferential direction. Suppose that the origin of the Cartesian coordinate system is selected at the scaling center of a sub-domain. Then the scaled boundary and Cartesian coordinate system of the sub-domain can be related by the scaling equations:

$$
x=\xi x_{\eta}(\eta)=\xi \mathbf{N}(\eta) \mathbf{x}_{s}, \quad y=\xi y_{\eta}(\eta)=\xi \mathbf{N}(\eta) \mathbf{y}_{s}
$$

where $x_{\eta}(\eta)$ and $y_{\eta}(\eta)$ describe the sub-domain boundary coordinates using continuous piecewise smooth functions; $\mathbf{x}_{s}$ and $\mathbf{y}_{s}$ describe the nodes' coordinate vectors of the sub-domain boundary; $\mathbf{N}(\eta)$ is the shape function. The displacements are expressed as the following form:

$$
\mathbf{u}(\xi, \eta)=\mathbf{N}^{u}(\eta) \mathbf{u}(\xi)
$$

where $\mathbf{u}(\xi)$ defines nodal displacement function along the radial lines, and $\mathbf{N}^{u}(\eta)$ is the shape function matrix at the circumferential direction.

The scaled boundary finite element equations can be derived according to the virtual work 
principle in elastic statics [32]:

$$
\begin{gathered}
\mathbf{p}_{s}=\mathbf{E}_{0} \frac{\partial \mathbf{u}(\xi)}{\partial \xi}+\left.\mathbf{E}_{1}^{T} \mathbf{u}(\xi)\right|_{\xi=1} \\
\mathbf{E}_{0} \xi^{2} \frac{\partial^{2} \mathbf{u}(\xi)}{\partial^{2} \xi}+\left[\mathbf{E}_{0}+\mathbf{E}_{1}^{T}-\mathbf{E}_{1}\right] \xi \frac{\partial \mathbf{u}(\xi)}{\partial \xi}-\mathbf{E}_{2} \mathbf{u}(\xi)=\mathbf{0}
\end{gathered}
$$

where $\mathbf{p}_{s}$ is the equivalent nodal force vector. The coefficient matrices $\mathbf{E}_{0}, \mathbf{E}_{1}$ and $\mathbf{E}_{2}$ are integrated along the sub-domain boundary $S$ :

$$
\begin{aligned}
& \mathbf{E}_{0}=\int_{S} \mathbf{B}_{1}^{T}(\eta) \mathbf{D B}_{1}(\eta)|J| d \eta \\
& \mathbf{E}_{1}=\int_{S} \mathbf{B}_{2}^{T}(\eta) \mathbf{D B}_{1}(\eta)|J| d \eta \\
& \mathbf{E}_{2}=\int_{S} \mathbf{B}_{2}^{T}(\eta) \mathbf{D B}_{2}(\eta)|J| d \eta
\end{aligned}
$$

where $\mathbf{D}$ is the elasticity matrix, $\mathbf{B}_{1}(\eta)$ and $\mathbf{B}_{2}(\eta)$ describe the strain-displacement relationship with:

$$
\begin{aligned}
& \mathbf{B}_{1}(\eta)=\frac{1}{|J|}\left[\mathbf{L}_{1} \frac{\partial y_{\eta}(\eta)}{\partial \eta}-\mathbf{L}_{2} \frac{\partial x_{\eta}(\eta)}{\partial \eta}\right] \mathbf{N}^{u}(\eta) \\
& \mathbf{B}_{2}(\eta)=\frac{1}{|J|}\left[-\mathbf{L}_{1} y_{\eta}(\eta)+\mathbf{L}_{2} x_{\eta}(\eta)\right] \frac{\partial \mathbf{N}^{u}(\eta)}{\partial \eta}
\end{aligned}
$$

where $\mathbf{L}_{1}$ and $\mathbf{L}_{2}$ are two linear operator matrices. $|J|$ is the determinant of the Jacobian matrix on the boundary:

$$
|J|=x_{\eta}(\eta) \frac{\partial y_{\eta}(\eta)}{\partial \eta}-y_{\eta}(\eta) \frac{x_{\eta}(\eta)}{\partial \eta}
$$

Since Eq. (4) is a second-order homogeneous Euler-Cauchy differential equation system with respect to $\xi$, it has the solution of the following form [32]:

$$
\mathbf{u}(\xi)=\sum_{i=1}^{n} c_{i} \xi^{-\lambda_{i}} \phi_{i}
$$

where $c_{i}$ is the integration constant, $\lambda_{i}$ is the modal scaling factor in the radial direction, $\phi_{i}$ is the modal displacement, and $n$ is the total degree of freedom of the sub-domain's nodes. Omitting the 
subscripts in Eq. (11), each displacement mode takes the form $\mathbf{u}(\xi)=\xi^{-\lambda} \phi$. Substituting this solution into Eqs. (3) and (4), and assembling together the two sets of equations leads to a linear eigenproblem:

$$
\mathbf{Z} \boldsymbol{\Phi}=\boldsymbol{\Phi} \boldsymbol{\lambda}, \quad \mathbf{Z}=\left[\begin{array}{ll}
\mathbf{Z}_{11} & \mathbf{Z}_{12} \\
\mathbf{Z}_{21} & \mathbf{Z}_{22}
\end{array}\right]=\left[\begin{array}{cc}
\mathbf{E}_{0}^{-1} \mathbf{E}_{1}^{T} & -\mathbf{E}_{0}^{-1} \\
\mathbf{E}_{1} \mathbf{E}_{0}^{-1} \mathbf{E}_{1}^{T}-\mathbf{E}_{2} & -\mathbf{E}_{1} \mathbf{E}_{0}^{-1}
\end{array}\right]
$$

where $\mathbf{Z}$ is a $2 n \times 2 n$ Hamilton matrix. There are $2 n$ modes in the solution of this standard eigenproblem, and for a bounded domain only the modes with non-positive real components of eigenvalues $\left(\left[\lambda_{1}, \lambda_{2}, \ldots, \lambda_{n}\right]\right)$ lead to finite displacements at the scaling centre. Therefore, the eigenvalues and eigenvectors in Eq. (12) are partitioned as follow:

$$
\mathbf{Z}\left[\begin{array}{ll}
\boldsymbol{\Phi}_{11} & \boldsymbol{\Phi}_{12} \\
\boldsymbol{\Phi}_{21} & \boldsymbol{\Phi}_{22}
\end{array}\right]=\left[\begin{array}{ll}
\boldsymbol{\Phi}_{11} & \boldsymbol{\Phi}_{12} \\
\boldsymbol{\Phi}_{21} & \boldsymbol{\Phi}_{22}
\end{array}\right]\left[\begin{array}{cc}
\lambda_{\mathrm{N}} & \mathbf{0} \\
\mathbf{0} & \lambda_{\mathrm{P}}
\end{array}\right]
$$

where $\lambda_{\mathrm{N}}$ and $\lambda_{\mathrm{P}}$ denote the negative and positive eigenvalue diagonal matrices, respectively. Then, the stiffness matrix of the sub-domain can be calculated by:

$$
\mathbf{K}_{e}=\Phi_{21} \Phi_{11}^{-1}
$$

The stiffness matrix of the whole domain can be obtained by assembling the stiffness matrices of all the sub-domains in exactly the same way as the FEM:

$$
\mathbf{K}=\sum_{i=1}^{N} \mathbf{G}_{i}^{T} \mathbf{K}_{e i} \mathbf{G}_{i}
$$

where $\Sigma$ stands for the matrix assembling rather than a simple sum operation, $N$ is the number of the sub-domains, $\mathbf{K}_{e i}$ is the stiffness matrix of the $i$ th sub-domain, and $\mathbf{G}_{i}$ is the transition matrix for the assembling corresponding to $\mathbf{K}_{e i}$.

The boundary nodal displacements $\mathbf{U}$ of the domain can be solved from the equilibrium equation after the displacement constraints and external loading conditions are imposed:

$$
\mathbf{K U}=\mathbf{p}
$$

where $\mathbf{p}$ is the global equivalent external nodal force vector which is assembled from $\mathbf{p}_{s}$ in Eq. (3). Suppose that $\mathbf{U}_{e}$ is the nodal displacement vector on the boundary of each sub-domain, and it 
can be extracted from $\mathbf{U}$. The integration constant matrix $\mathbf{c}$ of each sub-domain can be calculated by:

$$
\mathbf{c}=\boldsymbol{\Phi}_{11}^{-1} \mathbf{U}_{e}
$$

Then, the displacement field of each sub-domain is obtained as:

$$
\mathbf{u}(\xi, \eta)=\mathbf{N}^{u}(\eta) \sum_{i=1}^{n} c_{i} \xi^{-\lambda_{i}} \phi_{i}
$$

where $\phi_{i}$ is the ith column of $\boldsymbol{\Phi}_{11}$.

In addition, the stress field of each sub-domain is given as follows:

$$
\boldsymbol{\sigma}_{s}(\xi, \eta)=[\mathbf{D}] \sum_{i=1}^{n} c_{i} \xi^{-\lambda_{i}-1}\left[-\lambda_{i}\left[\mathbf{B}_{1}(\eta)\right]+\mathbf{B}_{2}(\eta)\right] \boldsymbol{\phi}_{i}
$$

\section{Formulation of the SSBFEM}

In this section, the formulation of SSBFEM is presented. The proposed method enables efficiently computing the stochastic structural responses under random field properties. It extends the deterministic SBFEM to a stochastic framework involving elements with random properties. This section mainly consists of four parts. First, the representation of the random field considering the spatial variability of material properties is given. Second, the stochastic equilibrium equation is formulated by introducing the random field into the polygon-based scaled boundary finite elements. Third, the perturbation method is employed to calculate the statistical moments of the stochastic structural responses. Finally, a sensitivity method is given to calculate the derivatives of the global stiffness matrix.

\subsection{Representation of the random field}

To represent the spatial variability of the material properties, the random field is generally employed. The random field is a collection of random variables with inter-correlations. As shown in Fig. 2, for a given point $\mathbf{x}_{0}$, the material property in this point is considered as a random variable. Therefore, in theory, the random field consists of an infinite number of random variables. For the consideration of the computational cost, the random field can normally be expressed using a finite number of random variables, and here the Karhunen-Loève (KL) expansion [23] is employed to 
address this problem. The Gaussian random field is adopted in many works (e.g., [23, 33]) for its simplicity and also as a result of the central limit theorem [29], and thus it is adopted in this paper. In addition, considering that the material properties appearing in the practical engineering problems are mostly non-negative [27], the positive random field (here the lognormal field) is also studied to model the randomly distributed material properties.

\subsubsection{The Karhunen-Loève (KL) expansion}

Among the various methods for the simulation of random fields, the KL expansion [23] is an optimal series expansion method. It is efficient and convenient to use since no mesh is required during the random field discretization, and therefore it is adopted in this paper. The KL expansion can be seen as a special case of the orthogonal series expansion where the orthogonal functions are chosen as the eigenfunctions of a Fredholm integral equation (IEVP) [27]. Suppose that $H(\mathbf{x}, \theta)$ is the random field, where $\mathbf{x}$ is the coordinate vector and $\theta$ is used to indicate that the involved quantities are random. When the Young's modulus is being modeled as a random field and $\theta=\left[\theta_{1}, \theta_{2}, \ldots, \theta_{Q}\right], Q$ random field properties can be then obtained, namely, $H\left(\mathbf{x}, \theta_{i}\right), i=1,2, \ldots Q$. In the case of Gaussian random field, it can be represented by the following expansion [23]:

$$
H(\mathbf{x}, \theta)=\mu(\mathbf{x})+\sum_{i=1}^{\infty} \sigma(\mathbf{x}) \sqrt{\gamma_{i}} \varsigma_{i}(\theta) \varphi_{i}(\mathbf{x})
$$

where $\mu(\mathbf{x})$ and $\sigma(\mathbf{x})$ denote the mean and standard deviation of the random field, respectively; $\left\{\varsigma_{i}(\theta), i=1,2,3, \ldots\right\}$ is a set of independent standard normal variables; $\gamma_{i}$ and $\varphi_{i}(\mathbf{x})$ are the $i$ th eigenvalue and eigenfunction, respectively, which are obtained from the following Fredholm integral equation [27]:

$$
\int_{\Omega} \operatorname{Cov}\left(\mathbf{x}, \mathbf{x}^{\prime}\right) \varphi_{i}\left(\mathbf{x}^{\prime}\right) \mathrm{d} \mathbf{x}^{\prime}=\gamma_{i} \varphi_{i}(\mathbf{x})
$$

where $\Omega$ is the spatial domain, $\mathbf{x}$ and $\mathbf{x}^{\prime}$ are two points in $\Omega \cdot \operatorname{Cov}\left(\mathbf{x}, \mathbf{x}^{\prime}\right)$ is the autocorrelation function which can be expressed as $\operatorname{Cov}\left(\mathbf{x}, \mathbf{x}^{\prime}\right)=\sigma(\mathbf{x}) \sigma\left(\mathbf{x}^{\prime}\right) \rho\left(\mathbf{x}, \mathbf{x}^{\prime}\right)$, in which $\rho\left(\mathbf{x}, \mathbf{x}^{\prime}\right)$ is the autocorrelation coefficient function. Analytical solutions of Eq. (21) can be obtained only for triangular and exponential autocorrelation function on a rectangular domain [23]. For random fields with arbitrary autocorrelation functions or domains of complex geometrical shapes, the 
eigensolutions need to be approximated numerically, and this point will be discussed in Section 3.1.2. In the practical calculation, the KL representation of random field is generally replaced by a truncated series expansion:

$$
\tilde{H}(\mathbf{x}, \theta)=\mu(\mathbf{x})+\sum_{i=1}^{M} \sigma(\mathbf{x}) \sqrt{\gamma_{i}} \varsigma_{i}(\theta) \varphi_{i}(\mathbf{x})
$$

where $M$ denotes the terms corresponding to the largest $M$ eigenvalues. For the case of lognormal field, it can be treated as the result of the exponentiation of a Gaussian random field [34]:

$$
\tilde{H}(\mathbf{x}, \theta)=\exp \left[\mu_{1}(\mathbf{x})+\sum_{i=1}^{M} \sigma_{1}(\mathbf{x}) \sqrt{\gamma_{i}} \varsigma_{i}(\theta) \varphi_{i}(\mathbf{x})\right]
$$

where the mean value $\mu_{1}(\mathbf{x})$ and standard deviation $\sigma_{1}(\mathbf{x})$ of the underlying Gaussian field are first computed by:

$$
\begin{gathered}
\sigma_{1}(\mathbf{x})=\sqrt{\operatorname{In}\left(1+\sigma^{2}(\mathbf{x}) / \mu^{2}(\mathbf{x})\right)} \\
\mu_{1}(\mathbf{x})=\operatorname{In} \mu(\mathbf{x})-\frac{1}{2} \sigma_{1}^{2}(\mathbf{x})
\end{gathered}
$$

As a result, the lognormal field can be approximated by Eq. (23).

\subsubsection{Numerical solutions of the IEVP}

The numerical approximation methods of the KL expansion mainly include the Nyström method [35], collocation method [34] or Galerkin method [24]. In this work, the Nyström method (EOLE) [36], which is based on the EOLE expansion [37], is adopted since it is straightforward to implement and no integration is required. It has been proved that the EOLE expansion is equivalent to the Nyström method when the integration points are uniformly distributed over the analyzed domain [36]. The basic steps of the Nyström method (EOLE) are described as follows:

(1) The points are uniformly distributed over the domain $\Omega$. This can be realized by sampling the uniform distribution over $\Omega$ or using the nodes of an equispaced structured grid.

(2) When the domain is not a simple shape, the integration procedure can be performed on a geometrically simpler primitive domain $\Omega^{*}$ that contains the physical domain $\Omega$.

(3) The points outside of $\Omega$ are not taken into consideration, and based on these points, the EOLE method is feasible to simulate the random field as a truly meshless method.

(4) By solving the eigenvalue problem of the autocorrelation coefficient matrix $\rho$, the following 
approximate KL expansion can be obtained:

$$
\begin{gathered}
\tilde{H}(\mathbf{x}, \theta)=\mu(\mathbf{x})+\sum_{i=1}^{M} \sigma(\mathbf{x}) \frac{\varsigma_{i}(\theta)}{\sqrt{\gamma_{i}^{*}}}\left[\varphi_{i}^{*}\right]^{T} \rho \quad \text { for Gaussian field } \\
\tilde{H}(\mathbf{x}, \theta)=\exp \left(\mu_{1}(\mathbf{x})+\sum_{i=1}^{M} \sigma_{1}(\mathbf{x}) \frac{\varsigma_{i}(\theta)}{\sqrt{\gamma_{i}^{*}}}\left[\varphi_{i}^{*}\right]^{T} \boldsymbol{\rho}\right) \text { for lognormal field }
\end{gathered}
$$

where $\gamma_{i}^{*}$ and $\varphi_{i}^{*}$ are the $i$ th eigenvalue and eigenvector of $\rho$, respectively. $\gamma_{i}^{*}$ is related to $\sqrt{\gamma_{i}}$ in Eq. (20) as $\gamma_{i}^{*}=\frac{N_{p}}{|\Omega|} \gamma_{i}$, where $N_{p}$ is the number of the integration points and $|\Omega|$ is the area of $\Omega$.

\subsection{Formulation of the stochastic equilibrium equation}

The stochastic response analysis under random field property is often used for the random non-homogenous problem, while it is challenging to use the SBFEM to deal with this kind of problem since a large number of sub-domains will be involved for the description of the spatial variability of the material property. Fortunately, Ooi et al. [14, 38] recently developed a polygon-based SBFEM which is able to discretize the analyzed domain into a number of polygons and thus the material property of each polygon is possible to be modeled independently, which provides an effective way to combine the SBFEM with the random field theory. Hence, to introduce the random field into the SBFEM equilibrium equation, the analyzed domain is first divided into a number of polygons. As shown in Fig. 3, the basic steps of the construction of the polygon mesh mainly include the discretization of the analyzed domain using Delaunay triangular mesh and the linkages between the centroids of all the triangular elements and the middle points. Through using the polygon mesh, an arbitrary domain can be flexibly dicretized into polygons which are modeled as sub-domains in SBFEM. For the case of a cracked domain, two kinds of polygon are included, namely, the non-cracked polygon and cracked polygon. For the non-cracked polygon, the scaling center is selected at its centroid, and for cracked polygon it is selected at the crack tip. More details about the scaled boundary polygon mesh can be referred to Ref. [38]. When there is a relatively strong correlation between the material properties in two adjacent points, it is desirable to consider the material property of each polygon as a random variable that can be defined as the value of the KL expansion at the scaling center $\overline{\mathbf{x}}$ of this polygon: 


$$
\begin{gathered}
\tilde{H}(\mathbf{x}, \theta)=\mu(\overline{\mathbf{x}})+\sum_{i=1}^{M} \sigma(\overline{\mathbf{x}}) \sqrt{\gamma_{i}} \varsigma_{i}(\theta) \varphi_{i}(\overline{\mathbf{x}}) \text { for Gaussian field } \\
\tilde{H}(\mathbf{x}, \theta)=\exp \left[\mu_{1}(\overline{\mathbf{x}})+\sum_{i=1}^{M} \sigma_{1}(\overline{\mathbf{x}}) \sqrt{\gamma_{i}} \varsigma_{i}(\theta) \varphi_{i}(\overline{\mathbf{x}})\right] \text { for lognormal field }
\end{gathered}
$$

Substituting Eq. (28) or Eq. (29) into the constitutive relation yields the random elasticity matrix:

$$
\mathbf{D}(\mathbf{x}, \theta)=\tilde{H}(\mathbf{x}, \theta) \mathbf{D}_{0}
$$

where $\mathbf{D}_{0}$ is the elasticity matrix computed with unit Young's modulus. Incorporating Eq. (30) into the coefficient matrices Eqs (5-7) of the SBFEM formulation leads to:

$$
\begin{aligned}
& \mathbf{E}_{0}(\theta)=\int_{S} \tilde{H}(\mathbf{x}, \theta) \mathbf{B}_{1}^{T}(\eta) \mathbf{D}_{0} \mathbf{B}_{1}(\eta)|J| d \eta \\
& \mathbf{E}_{1}(\theta)=\int_{S} \tilde{H}(\mathbf{x}, \theta) \mathbf{B}_{2}^{T}(\eta) \mathbf{D}_{0} \mathbf{B}_{1}(\eta)|J| d \eta \\
& \mathbf{E}_{2}(\theta)=\int_{S} \tilde{H}(\mathbf{x}, \theta) \mathbf{B}_{2}^{T}(\eta) \mathbf{D}_{0} \mathbf{B}_{2}(\eta)|J| d \eta
\end{aligned}
$$

where $\theta$ signifies that the coefficient matrices are random. Substituting Eqs. (31)-(33) into Eq. (12)yields the stochastic Hamilton matrix of a polygon:

$$
\mathbf{Z}(\theta)=\left[\begin{array}{cc}
\mathbf{E}_{0}^{-1}(\theta) \mathbf{E}_{1}^{T}(\theta) & -\mathbf{E}_{0}^{-1}(\theta) \\
\mathbf{E}_{1}(\theta) \mathbf{E}_{0}^{-1}(\theta) \mathbf{E}_{1}^{T}(\theta)-\mathbf{E}_{2}(\theta) & -\mathbf{E}_{1}(\theta) \mathbf{E}_{0}^{-1}(\theta)
\end{array}\right]
$$

Therefore, the stiffness matrix of each polygon is also a stochastic matrix rather than a deterministic one:

$$
\mathbf{K}_{e i}(\theta)=\Phi_{21}(\theta) \Phi_{11}^{-1}(\theta)
$$

Similarly, the above stochastic stiffness matrix in each polygon can be assembled into a global stochastic stiffness matrix:

$$
\mathbf{K}(\theta)=\sum_{i=1}^{N} \mathbf{G}_{i}^{T} \mathbf{K}_{e i}(\theta) \mathbf{G}_{i}
$$

where $\Sigma$ stands for the matrix assembling, and $N$ is the number of the total polygons (sub-domains). Finally, the nodal displacement vector $\mathbf{U}(\theta)$ of the whole domain can be solved by the following equilibrium equation:

$$
\mathbf{K}(\theta) \mathbf{U}(\theta)=\mathbf{p}
$$


For a given sample of the random field $\theta$, the above stochastic equilibrium equation can be solved by the deterministic solution procedure as the classical FEM. After solving Eq. (37), the nodal displacement vector $\mathbf{U}_{e}(\theta)$ of each polygon can be obtained from $\mathbf{U}(\theta)$. Combining Eqs (17-19), the stochastic displacements and stresses of the whole domain under random field can be calculated. It can be noted that a key feature of the SSBFEM is to insert the uncertainties into the coefficient matrices of the deterministic SBFEM as shown in Eqs. (31-33).

\subsection{The perturbation method for statistical analysis}

In order to calculate the statistical moments of the structural responses, the perturbation method [25] is employed. It is generally based on a Taylor series expansion of the quantities involved in the equilibrium equation. The basic variables used in the Taylor series expansion are $M$ independent standard normal variables $\varsigma_{j}, j=1,2, \cdots M$, which are employed to simulate the random field. The first order Taylor series expansions of $\mathbf{K}(\theta)$ and $\mathbf{U}(\theta)$ around their mean values can be expressed as:

$$
\begin{aligned}
& \mathbf{K}(\theta) \approx \mathbf{K}_{0}+\left.\sum_{j=1}^{M} \frac{\partial \mathbf{K}(\theta)}{\partial \varsigma_{j}}\right|_{\varsigma=\mathbf{0}} \varsigma_{j} \\
& \mathbf{U}(\theta) \approx \mathbf{U}_{0}+\left.\sum_{j=1}^{M} \frac{\partial \mathbf{U}(\theta)}{\partial \varsigma_{j}}\right|_{\zeta=\mathbf{0}} \varsigma_{j}
\end{aligned}
$$

where $\mathbf{K}_{0}$ and $\mathbf{U}_{0}$ are the mean parts of the stiffness matrix and displacement vector, respectively.

$$
\left.\frac{\partial \mathbf{K}(\theta)}{\partial \varsigma_{j}}\right|_{\varsigma=0} \text { and }\left.\frac{\partial \mathbf{U}(\theta)}{\partial \varsigma_{j}}\right|_{\varsigma=0} \text { represent the derivatives of } \mathbf{K}(\theta) \text { and } \mathbf{U}(\theta) \text { with respect to } \varsigma_{j}
$$

evaluated at $\boldsymbol{\varsigma}=\mathbf{0}$, respectively.

Substituting Eqs. (38) and (39) into Eq. (37) yields:

$$
\left(\mathbf{K}_{0}+\left.\sum_{j=1}^{M} \frac{\partial \mathbf{K}(\theta)}{\partial \varsigma_{j}}\right|_{\varsigma=0} \varsigma_{j}\right)\left(\mathbf{U}_{0}+\left.\sum_{j=1}^{M} \frac{\partial \mathbf{U}(\theta)}{\partial \varsigma_{j}}\right|_{\varsigma=0} \varsigma_{j}\right)=\mathbf{p}
$$

By identifying the coefficients of $\varsigma_{j}$ on both sides, one can obtain:

$$
\mathbf{U}_{0}=\mathbf{K}_{0}^{-1} \mathbf{p}
$$




$$
\frac{\partial \mathbf{U}(\theta)}{\partial \varsigma_{j}}=-\left.\mathbf{K}_{0}^{-1} \frac{\partial \mathbf{K}(\theta)}{\partial \varsigma_{j}}\right|_{\zeta=0} \mathbf{U}_{0}
$$

Finally, the mean vector and covariance matrix of $\mathbf{U}(\theta)$ are expressed as:

$$
\begin{gathered}
\mathrm{E}[\mathbf{U}(\theta)]=\mathbf{U}_{0} \\
\operatorname{Cov}[\mathbf{U}(\theta), \mathbf{U}(\theta)]=\left.\left.\sum_{j=1}^{M} \frac{\partial \mathbf{U}(\theta)}{\partial \varsigma_{j}}\right|_{\varsigma=0} \frac{\partial \mathbf{U}^{T}(\theta)}{\partial \varsigma_{j}}\right|_{\varsigma=0}
\end{gathered}
$$

From the Eqs (42) and (44), it can be known that before obtaining the structural response statistics, the gradients of the stiffness matrix under random field $\frac{\partial \mathbf{K}(\theta)}{\partial \varsigma_{j}}$ with respect to independent normal variables $\varsigma_{j}, j=1,2, \cdots M$, are required. It is thus important to obtain these sensitivity information efficiently and accurately.

\subsection{Derivatives of the global stiffness matrix}

The sensitivity analysis of the global stiffness matrix with respect to the uncertain parameters in the SSBFEM is a challenging task which is much more difficult than that in the classical SFEM, as the latter needs only to differentiate the stiffness matrix in a straightforward way. Recently, the authors [39] developed a sensitivity analysis method of SBFEM for elastostastics, through which the gradients of the structural responses with respect to the loads, material properties and geometrical characteristics can be semi-analytically calculated. Here, this method is extended to compute the gradients of the global stiffness matrix $\frac{\partial \mathbf{K}(\theta)}{\partial \varsigma_{j}}$ with respect to $\varsigma_{j}$ under random fields. $\frac{\partial \mathbf{K}(\theta)}{\partial \varsigma_{j}}$ can be assembled at the element level:

$$
\frac{\partial \mathbf{K}(\theta)}{\partial \varsigma_{j}}=\sum_{i=1}^{N} \mathbf{G}_{i}^{T} \frac{\partial \mathbf{K}_{e i}(\theta)}{\partial \varsigma_{j}} \mathbf{G}_{i}
$$

where $\Sigma$ represents the matrix assembling and $\frac{\partial \mathbf{K}_{e i}(\theta)}{\partial \varsigma_{j}}$ is the gradient of the $i$ th polygon's element stiffness matrix with respect to $\varsigma_{j}$, which can be computed by directly differentiating Eq. (35) with respect to $\varsigma_{j}$ : 


$$
\frac{\partial \mathbf{K}_{e i}(\theta)}{\partial \varsigma_{j}}=\left(\frac{\partial \Phi_{21}(\theta)}{\partial \varsigma_{j}}-\mathbf{K}_{e i}(\theta) \frac{\partial \Phi_{11}(\theta)}{\partial \varsigma_{j}}\right) \Phi_{11}^{-1}(\theta)
$$

From the above equation, it can be noted that before $\frac{\partial \mathbf{K}_{e i}(\theta)}{\partial \varsigma_{j}}$ is calculated, the gradients of the eigenvectors $\frac{\partial \Phi_{21}(\theta)}{\partial \varsigma_{j}}$ and $\frac{\partial \Phi_{11}(\theta)}{\partial \varsigma_{j}}$ of the polygon corresponding to $\mathbf{K}_{e i}(\theta)$ are required. To address this problem, we first focus on the gradient of the Hamilton matrix $\mathbf{Z}(\theta)$ with respect to $\varsigma_{j}$. Then the sensitivity analysis of the eigenproblem in Eq. (12) is performed, and whereby $\frac{\partial \Phi_{21}(\theta)}{\partial \varsigma_{j}}$ and $\frac{\partial \Phi_{11}(\theta)}{\partial \varsigma_{j}}$ are calculated.

\subsubsection{Derivatives of the Hamilton matrix}

The derivatives of the Hamilton matrix $\mathbf{Z}(\theta)$ with respect to $\varsigma_{j}$ can be calculated by differentiating Eq. (34):

$$
\frac{\partial \mathbf{Z}(\theta)}{\partial \varsigma_{j}}=\left[\begin{array}{ll}
\partial \mathbf{Z}_{11}(\theta) / \partial \varsigma_{j} & \partial \mathbf{Z}_{12}(\theta) / \partial \varsigma_{j} \\
\partial \mathbf{Z}_{21}(\theta) / \partial \varsigma_{j} & \partial \mathbf{Z}_{22}(\theta) / \partial \varsigma_{j}
\end{array}\right]
$$

where

$$
\begin{gathered}
\frac{\partial \mathbf{Z}_{11}(\theta)}{\partial \varsigma_{j}}=-\mathbf{E}_{0}^{-1}(\theta) \frac{\partial \mathbf{E}_{0}(\theta)}{\partial \varsigma_{j}} \mathbf{E}_{0}^{-1}(\theta) \mathbf{E}_{1}^{T}(\theta)+\mathbf{E}_{0}^{-1}(\theta) \frac{\partial \mathbf{E}_{1}^{T}(\theta)}{\partial \varsigma_{j}} \\
\frac{\partial \mathbf{Z}_{12}(\theta)}{\partial \varsigma_{j}}=\mathbf{E}_{0}^{-1}(\theta) \frac{\partial \mathbf{E}_{0}(\theta)}{\partial \varsigma_{j}} \mathbf{E}_{0}^{-1}(\theta) \\
\frac{\partial \mathbf{Z}_{21}(\theta)}{\partial \varsigma_{j}}=-\frac{\partial \mathbf{E}_{2}(\theta)}{\partial \varsigma_{j}}+\mathbf{E}_{1}(\theta) \frac{\partial \mathbf{Z}_{11}(\theta)}{\partial \varsigma_{j}}+\frac{\partial \mathbf{E}_{1}(\theta)}{\partial \varsigma_{j}} \mathbf{Z}_{11}(\theta) \\
\frac{\partial \mathbf{Z}_{22}(\theta)}{\partial \varsigma_{j}}=-\frac{\partial \mathbf{Z}_{11}^{T}(\theta)}{\partial \varsigma_{j}}
\end{gathered}
$$

where the derivatives of the coefficient matrices $\frac{\partial \mathbf{E}_{0}(\theta)}{\partial \varsigma_{j}}, \frac{\partial \mathbf{E}_{1}(\theta)}{\partial \varsigma_{j}}$ and $\frac{\partial \mathbf{E}_{2}(\theta)}{\partial \varsigma_{j}}$ can be performed at the element level before assembling the system. Substituting Eq. (28) into Eqs. (31) -(33), and differentiating them with respect to $\varsigma_{j}$ yields: 


$$
\begin{aligned}
& \frac{\partial \mathbf{E}_{0}(\theta)}{\partial \varsigma_{j}}=\sigma(\overline{\mathbf{x}}) \sqrt{\gamma_{j}} \int_{S} \varphi_{j}(\overline{\mathbf{x}}) \mathbf{B}_{1}^{T}(\eta) \mathbf{D}_{0} \mathbf{B}_{1}(\eta)|J| d \eta \\
& \frac{\partial \mathbf{E}_{1}(\theta)}{\partial \varsigma_{j}}=\sigma(\overline{\mathbf{x}}) \sqrt{\gamma_{j}} \int_{S} \varphi_{j}(\overline{\mathbf{x}}) \mathbf{B}_{2}^{T}(\eta) \mathbf{D}_{0} \mathbf{B}_{1}(\eta)|J| d \eta \\
& \frac{\partial \mathbf{E}_{2}(\theta)}{\partial \xi_{j}}=\sigma(\overline{\mathbf{x}}) \sqrt{\gamma_{j}} \int_{S} \varphi_{j}(\overline{\mathbf{x}}) \mathbf{B}_{2}^{T}(\eta) \mathbf{D}_{0} \mathbf{B}_{2}(\eta)|J| d \eta
\end{aligned}
$$

For the lognormal field, $\frac{\partial \mathbf{E}_{0}(\theta)}{\partial \varsigma_{j}}, \frac{\partial \mathbf{E}_{1}(\theta)}{\partial \varsigma_{j}}$ and $\frac{\partial \mathbf{E}_{2}(\theta)}{\partial \varsigma_{j}}$ can be simply obtained by substituting Eq. (29) into Eqs. (31)-(33), and then differentiating them with respect to $\varsigma_{j}$ :

$$
\begin{aligned}
& \frac{\partial \mathbf{E}_{0}(\theta)}{\partial \varsigma_{j}}=\exp \left(\mu_{1}\right) \sigma_{1}(\overline{\mathbf{x}}) \sqrt{\gamma_{j}} \int_{S} \varphi_{j}(\overline{\mathbf{x}}) \mathbf{B}_{1}^{T}(\eta) \mathbf{D}_{0} \mathbf{B}_{1}(\eta)|J| d \eta \\
& \frac{\partial \mathbf{E}_{1}(\theta)}{\partial \varsigma_{j}}=\exp \left(\mu_{1}\right) \sigma_{1}(\overline{\mathbf{x}}) \sqrt{\gamma_{j}} \int_{S} \varphi_{j}(\overline{\mathbf{x}}) \mathbf{B}_{2}^{T}(\eta) \mathbf{D}_{0} \mathbf{B}_{1}(\eta)|J| d \eta \\
& \frac{\partial \mathbf{E}_{2}(\theta)}{\partial \xi_{j}}=\exp \left(\mu_{1}\right) \sigma_{1}(\overline{\mathbf{x}}) \sqrt{\gamma_{j}} \int_{S} \varphi_{j}(\overline{\mathbf{x}}) \mathbf{B}_{2}^{T}(\eta) \mathbf{D}_{0} \mathbf{B}_{2}(\eta)|J| d \eta
\end{aligned}
$$

For the case of the random field with complex geometry, non-triangular and non-exponential autocorrelation function, Eq. (26) or Eq. (27) is used to obtain the approximate KL expansion. Following the same procedure with the above derivation, $\frac{\partial \mathbf{E}_{0}(\theta)}{\partial \varsigma_{j}}, \frac{\partial \mathbf{E}_{1}(\theta)}{\partial \varsigma_{j}}$ and $\frac{\partial \mathbf{E}_{2}(\theta)}{\partial \varsigma_{j}}$ can be calculated as well.

\subsubsection{Solution of the eigensensitivity problem}

The eigenvectors $\boldsymbol{\Phi}_{i}, i=1,2, \ldots, 2 n$ are usually not unique since they shall still satisfy Eq. (12) when multiplied or divided by any scalar. To address the sensitivity analysis of the eigenproblem in Eq. (12), a normalization condition is applied to ensure the uniqueness of the eigenvectors [40, 41]:

$$
\left\{\Phi_{i}(\theta)\right\}_{n_{i}}=1
$$

where $\boldsymbol{\Phi}_{i}(\theta)$ is the $i$ th column of $\boldsymbol{\Phi}(\theta)$, and $\left\{\bullet_{i}\right\}_{n_{i}}$ denotes its $n_{i}$ th component where $n_{i}$ is selected so that the $n_{i}$ th component in $\Phi_{i}(\theta)$ is the largest in absolute. Differentiating the 
eigenproblem in Eq. (12) with respect to $\varsigma_{j}$ leads to:

$$
\left(\mathbf{Z}(\theta)-\lambda_{i}(\theta) \mathbf{I}\right) \frac{\partial \boldsymbol{\Phi}_{i}(\theta)}{\partial \varsigma_{j}}=\frac{\partial \lambda_{i}(\theta)}{\partial \varsigma_{j}} \boldsymbol{\Phi}_{i}(\theta)-\frac{\partial \mathbf{Z}(\theta)}{\partial \varsigma_{j}} \boldsymbol{\Phi}_{i}(\theta)
$$

where I is a $2 n \times 2 n$ identity matrix. The derivatives of eigenvectors cannot be solved directly by using Eq. (59) since $\left(\mathbf{Z}(\theta)-\lambda_{i}(\theta) \mathbf{I}\right)$ is a singular matrix. To deal with this problem, an additional constraint is constructed by differentiating the normalization condition in Eq. (58) [42]:

$$
\left\{\frac{\partial \Phi_{i}(\theta)}{\partial \varsigma_{j}}\right\}_{n_{i}}=0 \text { or } \mathbf{W}_{i} \frac{\partial \Phi_{i}(\theta)}{\partial \varsigma_{j}}=0, i=1,2, \ldots n
$$

where $\mathbf{W}_{i}$ is a vector associated with the $i$ th eigenvector, and all the components of it are zeros except for the $n_{i}$ th component that is set to unity. The sensitivity analysis of the eigenvalues and eigenvectors can be then achieved by solving the following linear system constructed by combining Eqs. (59) and (60):

$$
\left[\begin{array}{cc}
\mathbf{Z}(\theta)-\lambda_{i}(\theta) \mathbf{I} & -\boldsymbol{\Phi}_{i}(\theta) \\
\mathbf{W}_{i} & 0
\end{array}\right]\left[\begin{array}{c}
\frac{\partial \boldsymbol{\Phi}_{i}(\theta)}{\partial \varsigma_{j}} \\
\frac{\partial \lambda_{i}(\theta)}{\partial \varsigma_{j}}
\end{array}\right]=\left[\begin{array}{c}
-\frac{\partial \mathbf{Z}(\theta)}{\partial \varsigma_{j}} \boldsymbol{\Phi}_{i}(\theta) \\
0
\end{array}\right]
$$

where $\frac{\partial \mathbf{Z}(\theta)}{\partial \varsigma_{j}}$ can be obtained from Eq. (47). The numerical stability of the above equations have been proved in Ref. [43]. By solving the above equations, $\frac{\partial \lambda_{i}(\theta)}{\partial \varsigma_{j}}$ and $\frac{\partial \Phi(\theta)}{\partial \varsigma_{j}}$ can be calculated, and $\frac{\partial \Phi(\theta)}{\partial \varsigma_{j}}$ is rewritten as:

$$
\frac{\partial \Phi(\theta)}{\partial \varsigma_{j}}=\left[\begin{array}{ll}
\frac{\partial \Phi_{11}(\theta)}{\partial \varsigma_{j}} & \frac{\partial \Phi_{12}(\theta)}{\partial \varsigma_{j}} \\
\frac{\partial \Phi_{21}(\theta)}{\partial \varsigma_{j}} & \frac{\partial \Phi_{22}(\theta)}{\partial \varsigma_{j}}
\end{array}\right]
$$

Through the above equation, $\frac{\partial \Phi_{11}(\theta)}{\partial \varsigma_{j}}$ and $\frac{\partial \Phi_{21}(\theta)}{\partial \varsigma_{j}}$ can be obtained. Substituting them into Eq. 
(46), $\frac{\partial \mathbf{K}_{e i}(\theta)}{\partial \varsigma_{j}}$ can be then obtained. Finally, the derivative of the global stiffness matrix $\frac{\partial \mathbf{K}(\theta)}{\partial \varsigma_{j}}$ can be assembled, and combining Eqs. (42)-(44), the mean and covariance matrices of $\mathbf{U}(\theta)$ are further calculated.

\section{Application to stochastic fracture mechanics}

In this section, the proposed SSBFEM is applied to the stochastic fracture mechanics analysis under randomly distributed material properties. To express the singularity of the crack tip, the stress intensity factors (SIFs) $K$ are introduced by Irwin [44], which are partitioned to $K_{\mathrm{I}}$ and $K_{\mathrm{II}}$ in plane problems. In the SBFEM, the SIFs can be directly extracted from the semi-analytical solutions of the displacements [31] or the stresses [9]. Here, the latter is adopted to calculate the SIFs. In the case of random field properties, the stress solution in Eq. (19) can be expressed as:

$$
\sigma_{s}(\xi, \eta, \theta)=\sum_{i=1}^{n} c_{i}(\theta) \xi^{-\lambda_{i}(\theta)-1} \Gamma_{i}(\theta)
$$

where

$$
\Gamma_{i}(\theta)=\left\{\begin{array}{c}
\Gamma_{x x}(\theta) \\
\Gamma_{y y}(\theta) \\
\Gamma_{x y}(\theta)
\end{array}\right\}_{i}=\mathbf{D}(\theta)\left[-\lambda_{i}(\theta) \mathbf{B}_{1}(\eta)+\mathbf{B}_{2}(\eta)\right] \phi_{i}(\theta)
$$

Figure 4 shows the cracked polygon modeled by the SBFEM and the coordinate origin $O$ is set at the crack tip. For the reason that the material property in a polygon is considered to be homogeneous, the singular stresses generated at the crack tip mainly consist of two singular stress terms, denoted as I and II. Under such circumstance, one can obtain $-1<\lambda_{i}<0$. Hence, the SIFs can be expressed as follow [45]:

$$
\left\{\begin{array}{l}
K_{\mathrm{I}}(\theta) \\
K_{\mathrm{II}}(\theta)
\end{array}\right\}=\sqrt{2 \pi L_{0}}\left\{\begin{array}{l}
\sum_{i=I, I I} c_{i}(\theta)\left\{\Gamma_{y y}(\theta)\right\}\left(\eta=\eta_{A}\right)_{i} \\
\sum_{i=I, I I} c_{i}(\theta)\left\{\Gamma_{x y}(\theta)\right\}\left(\eta=\eta_{A}\right)_{i}
\end{array}\right\}
$$

where $L_{0}$ is the distance between the crack tip and the boundary along the crack extension line, and 
$\eta_{A}$ is the circumferential coordinate of the intersection of the extension line and the boundary of the cracked polygon.

Since the material property of the structure is randomly distributed across the space, uncertainty also exists in the SIFs. By introducing the Taylor series expansion, the first-order approximations of the SIFs around their mean values can be expressed as:

$$
\left\{\begin{array}{l}
K_{\mathrm{I}}(\theta) \\
K_{\mathrm{II}}(\theta)
\end{array}\right\}=\left\{\begin{array}{l}
\bar{K}_{\mathrm{I}}+\left.\sum_{j=1}^{M} \frac{\partial K_{\mathrm{I}}(\theta)}{\partial \varsigma_{j}}\right|_{\varsigma=0} \varsigma_{j} \\
\bar{K}_{\mathrm{II}}+\left.\sum_{j=1}^{M} \frac{\partial K_{\mathrm{II}}(\theta)}{\partial \varsigma_{j}}\right|_{\zeta=0} \varsigma_{j}
\end{array}\right\}
$$

where $\bar{K}_{\mathrm{I}}$ and $\bar{K}_{\mathrm{II}}$ are the mean part of the SIFs, $\left.\frac{\partial K_{\mathrm{I}}(\theta)}{\partial \varsigma_{j}}\right|_{\varsigma=0}$ and $\left.\frac{\partial K_{\mathrm{II}}(\theta)}{\partial \varsigma_{j}}\right|_{\varsigma=0}$ are the derivatives of $K_{\mathrm{I}}(\theta)$ and $K_{\mathrm{II}}(\theta)$ with respect to $\varsigma_{j}$ evaluated at $\boldsymbol{\varsigma}=\mathbf{0}$, respectively. According to Eq. (66), the first order estimation of the means and variances of the SIFs can be obtained:

$$
\begin{gathered}
\left\{\begin{array}{l}
\mathrm{E}\left[K_{\mathrm{I}}(\theta)\right] \\
\mathrm{E}\left[K_{\mathrm{II}}(\theta)\right]
\end{array}\right\}=\left\{\begin{array}{l}
\bar{K}_{\mathrm{I}} \\
\bar{K}_{\mathrm{II}}
\end{array}\right\} \\
\left\{\begin{array}{l}
\operatorname{Var}\left[K_{\mathrm{I}}(\theta)\right] \\
\operatorname{Var}\left[K_{\mathrm{II}}(\theta)\right]
\end{array}\right\}=\left\{\begin{array}{l}
\left.\sum_{j=1}^{M} \frac{\partial K_{\mathrm{I}}^{2}(\theta)}{\partial \varsigma_{j}}\right|_{\zeta=0} \\
\left.\sum_{j=1}^{M} \frac{\partial K_{\mathrm{II}}^{2}(\theta)}{\partial \varsigma_{j}}\right|_{\varsigma=0}
\end{array}\right\}
\end{gathered}
$$

The above equations indicate that in order to calculate the statistical characteristics of the SIFs, it is still necessary to calculate the sensitivities of the SIFs with respect to $\varsigma_{j}$. Differentiating Eq. (65) yields:

$$
\left\{\begin{array}{l}
\frac{\partial K_{\mathrm{I}}(\theta)}{\partial \varsigma_{j}} \\
\frac{\partial K_{\mathrm{II}}(\theta)}{\partial \varsigma_{j}}
\end{array}\right\}=\sqrt{2 \pi L_{0}}\left\{\begin{array}{l}
\sum_{i=I, I I} \frac{\partial c_{i}(\theta)}{\partial \varsigma_{j}} \Gamma_{y y}\left(\theta, \eta=\eta_{A}\right)_{i}+c_{i}(\theta) \frac{\partial \Gamma_{y y}\left(\theta, \eta=\eta_{A}\right)_{i}}{\partial \varsigma_{j}} \\
\sum_{i=I, I I} \frac{\partial c_{i}(\theta)}{\partial \varsigma_{j}} \Gamma_{x y}\left(\theta, \eta=\eta_{A}\right)_{i}+c_{i}(\theta) \frac{\partial \Gamma_{x y}\left(\theta, \eta=\eta_{A}\right)_{i}}{\partial \varsigma_{j}}
\end{array}\right\}
$$

where $\frac{\partial c_{i}(\theta)}{\partial \varsigma_{j}}$ is the derivative of the integration constant $c_{i}(\theta)$ with respect to $\varsigma_{j}$, which can 
be obtained from the integration constant vector $\frac{\partial \mathbf{c}}{\partial \varsigma_{j}}$ calculated by differentiating Eq. (17) with respect to $\varsigma_{j}$ :

$$
\frac{\partial \mathbf{c}(\theta)}{\partial \varsigma_{j}}=\boldsymbol{\Phi}_{11}^{-1}(\theta)\left(\frac{\partial \mathbf{U}_{i}(\theta)}{\partial \varsigma_{j}}-\frac{\partial \Phi_{11}^{-1}(\theta)}{\partial \varsigma_{j}} \mathbf{c}(\theta)\right)
$$

where $\frac{\partial \mathbf{U}_{i}(\theta)}{\partial \varsigma_{j}}$ is the derivative of the nodal displacement vector of the $i$ th polygon with respect to $\varsigma_{j}$, which can be fetched from $\frac{\partial \mathbf{U}(\theta)}{\partial \varsigma_{j}}$ calculated by Eq. (42). In addition, $\frac{\partial \Gamma_{y y}(\theta)}{\partial \varsigma_{j}}$ and $\frac{\partial \Gamma_{x y}(\theta)}{\partial \varsigma_{j}}$ can be derived by differentiating Eq. (64):

$$
\begin{aligned}
& \frac{\partial \boldsymbol{\Gamma}_{i}(\theta)}{\partial \varsigma_{j}}=\frac{\partial \mathbf{D}(\theta)}{\partial \varsigma_{j}} \sum_{i=1}^{n}\left(-\lambda_{i}(\theta) \mathbf{B}_{1}+\mathbf{B}_{2}\right) \boldsymbol{\phi}_{i}(\theta)+ \\
& \mathbf{D}(\theta) \sum_{i=1}^{n}\left[-\frac{\partial \lambda_{i}(\theta)}{\partial \varsigma_{j}} \mathbf{B}_{1} \phi_{i}(\theta)+\mathrm{c}_{i}(\theta)\left(-\lambda_{i}(\theta) \mathbf{B}_{1}+\mathbf{B}_{2}\right) \frac{\partial \boldsymbol{\phi}_{i}(\theta)}{\partial \varsigma_{j}}\right]
\end{aligned}
$$

where

$$
\begin{gathered}
\frac{\partial \mathbf{D}(\theta)}{\partial \varsigma_{j}}=\sigma\left(\overline{\mathbf{x}}^{*}\right) \sqrt{\gamma_{j}} \varphi_{j}\left(\overline{\mathbf{x}}^{*}\right) \mathbf{D}_{0} \text { for Gaussian field } \\
\frac{\partial \mathbf{D}(\theta)}{\partial \varsigma_{j}}=\exp \left(\mu_{1}\right) \sigma\left(\overline{\mathbf{x}}^{*}\right) \sqrt{\gamma_{j}} \varphi_{j}\left(\overline{\mathbf{x}}^{*}\right) \mathbf{D}_{0} \text { for lognormal field }
\end{gathered}
$$

where $\overline{\mathbf{x}}^{*}$ is located at the crack tip of the cracked polygon. As a result, all the gradients of the SIFs can be determined, and the means and variances of the SIFs under the random field properties can be further calculated using Eqs (67) and (68).

Though the considered random field involves only a single parameter in this work, i.e., the Young's modulus, the SSBFEM is easy to be extended to the scenario when the random field involves two or more independent parameters, such as the load, strength or fracture toughness in addition to the Young's modulus. For example, suppose that a response function with random fields involves two variables:

$$
F(\mathbf{x}, \theta)=R(\mathbf{x}, \theta)-\sigma_{s}(H(\mathbf{x}, \theta))
$$


where $\sigma_{s}$ denotes random field equivalent stress under random field material property $H(\mathbf{x}, \theta)$ and $R(\mathbf{x}, \theta)$ denotes the random field tensile strength:

$$
R(\mathbf{x}, \theta)=\mu^{\prime}(\mathbf{x})+\sum_{i=1}^{M^{\prime}} \sigma^{\prime}(\mathbf{x}) \sqrt{\gamma_{i}^{\prime}} \varsigma_{i}^{\prime}(\theta) \varphi_{i}^{\prime}(\mathbf{x})
$$

where $\mu^{\prime}(\mathbf{x})$ and $\sigma^{\prime}(\mathbf{x})$ denote the mean and standard deviation of the random field tensile strength. Substituting Eqs. (22) and (75) into Eq. (74), $F$ can be expressed as a function whose random variables are $\varsigma_{i}^{\prime}, i=1,2, \ldots, M^{\prime}$ and $\varsigma_{j}, j=1,2, \ldots, M$. The only difference between this scenario and that illustrated in this paper is that here the required random variables are more, but the treatment is almost the same. The sensitivity analysis of the stresses can be referred to Ref. [39]. If the two random fields are dependent, the random fields should be first transformed to be independent. Then the problem could become complicated.

\section{Numerical examples}

\subsection{The cantilever beam}

Figure 5(a) shows a two-dimensional cantilever beam with dimensions $L=10$ units and $D=2$ units. The left edge is fixed and the right edge is subjected to a shear load $p=150$ units . The Young's modulus $E$ of the plate is modeled as Gaussian random field with the mean $\mu=3 \mathrm{e} 7$ units and the standard variance $\sigma=3 \mathrm{e} 6$ units . Poisson's ratio $v$ is equal to 0.25 . The plane stress condition is assumed. In the polygon mesh, there are a total of 146 polygons and 338 nodes as shown in Fig. 5(b). The autocorrelation function is assumed to be the exponential type. The correlation length along the $x$ and $y$ directions are set to be 7 units and 1 unit, respectively. The SSBFEM is used to calculate the standard deviation of the displacement of node $\mathrm{C}$ in the $y$ direction. To investigate the effects of the order $M$ of the KL expansion on the results, $M$ is set to be variable from 1 to 8 and the standard deviation is calculated for each value of $M$. As shown in Fig. 6, the results are converged when $M$ is more than 4, and thus $M$ is set to be 6 in the following stochastic response analysis. 
Table 1 shows the means and standard deviations of the displacements of the polygon corresponding to node $\mathrm{C}$, where the nodes of the polygon are anti-clockwise distributed and node $\mathrm{C}$ is the endpoint. To verify the computational accuracy of the proposed approach, the results of the MCS using 10000 samples are chosen as the reference solution. It can be noted that the two results given by the two methods agree very well with the largest relative error of the means and standard deviations equal to $0.69 \%$ and $1.74 \%$, respectively. However, the MCS requires 10000 evaluations of the SBFEM, while the SSBFEM needs only one evaluation of the SBFEM at the mean value of the random field and the calculation of the semi-analytical sensitivities. It reduces the computational costs remarkably. Moreover, the means and standard deviations of the whole displacements of the analyzed domain are depicted in Fig. 7. From the results of the means, it can be found that for this problem, the displacement distribution of the plate under randomly distributed material property is similar to the one under deterministic constant material property. From the results of the standard deviations, it can be known that the displacements of each node are actually fluctuated, and the greatest standard deviations appear on the upper and lower edge of the plate in the $x$ direction and at the right part of the plate in the $y$ direction.

\subsection{The horizontal edge-cracked plate}

Figure 8(a) shows a rectangular flat plate with dimensions $2 L=2$ units and $W=1$ unit, and crack length $a=0.5$ unit . The analyzed domain is discretized into 242 polygons and 551 nodes as shown in Fig. 8(b). The Young's modulus $E$ of the plate is modeled as lognormal field with $\mu=20.7 \times 10^{6}$ units and $\sigma=4.14 \times 10^{6}$, and Poisson's ratio $v$ is equal to 0.3 . To guarantee the computational accuracy of the SIFs, the edges on the crack polygon are further subdivided into five linear elements [38]. The autocorrelation function is assumed to be the exponential type. The correlation length along the $x$ and $y$ directions are set to be 7 units and 1 unit, respectively. To calculate the statistical moments of the SIFs under the random field property, the derivatives of the SIFs with respect to the normal random variables $\varsigma_{j}$ for representing the random field are very important, as shown in Eq. (68). The accuracy of these derivatives is verified by using the central difference method. For example, the derivatives of $K_{\mathrm{I}}$ with respect to $\varsigma_{j}$ can be calculated as: 


$$
\frac{\partial K_{\mathrm{I}}}{\partial \varsigma_{j}}=\frac{K_{\mathrm{I}}\left(\varsigma_{1}, \ldots, \varsigma_{j}+\frac{1}{2} \delta \varsigma_{j}, \ldots, \varsigma_{M}\right)-K_{\mathrm{I}}\left(\varsigma_{1}, \ldots, \varsigma_{j}-\frac{1}{2} \delta \varsigma_{j}, \ldots, \varsigma_{M}\right)}{\delta \varsigma_{j}}
$$

where $\delta \varsigma_{j}$ is the perturbation of $\varsigma_{j}$ at a finite order of magnitude, and here 0.01 is selected. The results of the proposed sensitivity analysis method and the central difference method are given in Table 2. It shows that the two results agree very well with a maximum relative error only equal to $1.03 \%$. However, the central difference method requires $2 M+1$ evaluations of the SBFEM and its computational accuracy depends on difference step of the parameters. In contrast, the proposed sensitivity analysis method needs only one evaluation of the SBFEM and has no problem of the step's selection.

Since the gradients of SIFs with respect to $\zeta_{i}$ are obtained, the statistical moments of the SIFs can then be calculated by Eqs. (67) and (68). Figure 9 shows the standard deviations of $K_{\mathrm{I}}$ with $M$ varied from 1-18. The results show that the converged solution is obtained when $M$ is greater than 14 , which is much greater than the order 4 required in example 1. It indicates that the statistical analysis of the SIFs requires more orders of KL expansion than the one of the displacements. Thus, in the following analysis of this example, $M$ is selected to be 16 in order to guarantee the convergence of the results. Figure 10 shows the means and standard deviations of the whole displacements in the domain. It indicates that the largest fluctuated area of the displacements is mainly located at the middle of the plate in the $x$ direction, and at the left part of the plate in the $y$ direction. Table 3 gives the means and standard deviations of the SIFs under spatially varying random material property using both SSBFEM and MCS with 3000 samples. It can be found that the results obtained by the two methods show good agreement with the maximum relative error equal to $1.05 \%$. However, since that the random field property has been directly inserted into the representation of the SIFs and their sensitivities in the SSBFEM, the means and variances of the SIFs can be calculated efficiently by calling the SBFEM once, which saves computational costs significantly. Though both $K_{\mathrm{I}}$ and $K_{\mathrm{II}}$ are considered in this example, as shown in Table 3, the mean and standard deviation of $K_{\mathrm{II}}$ are close to zero, thus this example is still a quasi mode-I fracture problem. It indicates that the spatial variability of the material property may not affect the mode of fracture. 


\subsection{The inclined Edge-cracked plate}

A rectangular flat plate with dimensions $2 L=2$ units and $2 L=1$ unit is shown in Fig. 11(a). The upper and lower edges of the plate are subjected to a tensile stress with $\sigma=1$ unit. There is an inclined crack with dimensions $a=0.5$ unit and crack depth $z=0.5$ unit. The Young's modulus $E$ of the plate is modeled as lognormal field with $\mu=20.7 \times 10^{6}$ units and $\sigma=2.07 \times 10^{6}$ units. Poisson's ratio is equal to 0.3 . The analyzed domain is discretized into 250 polygons and 571 nodes as shown in Fig. 11(b). The autocorrelation function is assumed to be the exponential type. The correlation length in the $x$ and $y$ directions are assumed to 0.5 unit and 1.5 units, respectively. Figure 12 gives three arbitrary samples of random fields representing the spatial variability of the material property. It can be noted that the material spatial distribution of each sample is different and with random characteristic. Actually, this spatial variability of the material property can effectively affect the values of the SIFs and it is the reason why it is interesting to carry out the stochastic fracture analysis.

Before the statistical moments of the SIFs are obtained, the derivatives of the SIFs with respect to the normal variables $\varsigma_{i}$ are calculated both using the proposed sensitivity analysis method and the central difference method, as shown in Table 4. As with the above examples, the two results show good agreement with the maximum relative error equal to $1.75 \%$. The proposed sensitivity analysis method is used to calculate the standard derivations of the SIFs where the order $M$ is varied from 1 to 18 as depicted in Fig. 13. Similar to example 5.2, the results show that the converged solutions are obtained when $M$ is more than 14 and thus $M$ is set to be 16 in the following analysis. Table 5 shows the means and standard derivations of the SIFs using SSBFEM and MCS with 3000 samples. It can be seen that the two results have the maximum relative errors equal to $0.32 \%$ and $0.53 \%$ for $K_{\mathrm{I}}(\theta)$ and $K_{\mathrm{II}}(\theta)$, respectively, while the SSBFEM needs much less computational time. The examples 2 and 3 demonstrate that the SSBFEM allows dealing with both the mode-I and mixed-mode stochastic fracture problems.

\subsection{The edge-cracked plate with a hole}

An edge cracked plate with a circular hole of radius $R=3.45 \mathrm{~mm}$ is shown in Fig. 14(a). A uniform tension $p=6 \mathrm{MPa}$ is applied on the upper and lower edges of the plate. The Young's 
modulus $E$ of the plate is modeled as Gaussian random field with $\mu=200 \mathrm{MPa}$ and $\sigma=20 \mathrm{MPa}$, and Poisson's ratio $v=0.3$. In the polygon mesh, there are a total of 373 polygons and 852 nodes as shown in Fig. 14(b). Since the shape of plate is not a simple rectangle, numerical methods are required to solve the IEVP during the KL expansion. When using the Nyström method (EOLE), the integration points are distributed uniformly over the primitive domain as shown in Fig. 15(a). Both the Nyström method (EOLE) and the analytical method are performed for the IEVP in the case of primitive domain with rectangular geometry for the validation of the Nyström method (EOLE). Table 6 shows that the eigenvalues obtained by the two methods achieve good agreement with the maximum relative error equal to $5.34 \%$, which validates the performance of the Nyström method (EOLE). Then the solution of the IEVP for the physical domain is performed, where the points located outside the physical domain are not considered in the analysis as shown in Fig. 15(b). By considering Eq. (26) simultaneously, the random fields with the non-rectangular geometry shown in Fig. 14(a) can be simulated. The discretization of the random field is converged with respect to an increasing number of points. Figure 16 shows three arbitrary selected Gaussian random fields which have different material property distributions in the plate.

The SSBFEM is used to calculate the statistical moments of the SIFs. Since $K_{\text {II }}$ is close to zero, only $K_{\mathrm{I}}$ is considered. Figure 17 shows the standard deviation of $K_{\mathrm{I}}$ with $M$ varied from 1 to 18 . Similarly, the results appear to be converged when $M$ is greater than 14 , and thus 16 truncated terms are used in the following calculation. The MCS using 3000 samples is performed in order to verify the accuracy of the SSBFEM, and the results are shown in Table 7. The results obtained by the two methods agree well with the maximum relative error equal to $0.76 \%$, which indicates that the SSBFEM is applicable for the structures with non-rectangular geometries and thus is possible to be used for the stochastic fracture analysis of structures with complex geometries under randomly distributed material properties. Figure 18 depicts the global means and standard deviations of the whole displacements in the domain. It shows that the largest means of the displacements mainly distribute at the right side of the hole in the $x$ direction, while at the top of the hole in the $y$ direction. The areas of the largest standard deviations of the displacements in the $x$ direction include two parts: a strip across the hole and an area near the crack, while those in the $y$ direction 
mainly distribute at the bottom of the crack.

\section{Conclusion}

This paper proposes a SSBFEM to efficiently calculate the statistical characteristics of the structural responses considering the spatial variability of material properties. Through inserting the random field represented by the KL expansion into the constitutive relationship of the SBFEM, the stochastic equilibrium equation is formulated, which involves polygon elements with random material properties. The gradients of the global stiffness matrix with respect to the random variables for simulating the random fields are calculated semi-analytically. In particular, the SSBFEM is possessed of a capability on treating the stochastic fracture problems under random field property. Numerical examples are presented to illustrate the stochastic fracture behaviors of different types of cracked structures considering both rectangular and non-rectangular geometries. Through the SSBFEM, the mean values and fluctuation levels of the global structural responses are successfully estimated. The results show that the orders of the KL expansion required for the converged standard deviations of the SIFs are greater than those of the displacements; the statistical moments of the responses obtained by the SSBFEM agree well with those of the MCS but the former allows saving computational cost remarkably. In the future, the present method is possible to be applied to the statistical analysis of the concrete material or composite material problems. Moreover, it can also be extended to some other interesting topics such as the reliability analysis of the cracked structures and the optimization design on the distributions of the material properties under random fields.

\section{Acknowledgement}

This work is supported by the National Science Foundation of China (11172096), the State Key Program of National Science Foundation of China (11232004), the Funds for Distinguished Young Scientists of Hunan Province (14JJ1016), and the Graduate Student Research Innovation Project in Hunan Province (521293163). 


\section{References}

[1] C.M. Song, J.P. Wolf, The scaled boundary finite-element method—alias consistent infinitesimal finite-element cell method-for elastodynamics, Computer methods in applied mechanics and engineering, 147 (1997) 329-355.

[2] C.M. Song, J.P. Wolf, The scaled boundary finite-element method: analytical solution in frequency domain, Computer methods in applied mechanics and engineering, 164 (1998) 249-264.

[3] J.P. Wolf, Response of unbounded soil in scaled boundary finite-element method, Earthquake engineering \& structural dynamics, 31 (2002) 15-32.

[4] C. Birk, R. Behnke, A modified scaled boundary finite element method for three-dimensional dynamic soil-structure interaction in layered soil, International journal for numerical methods in engineering, 89 (2012) 371-402.

[5] M.H. Bazyar, A. Talebi, Transient seepage analysis in zoned anisotropic soils based on the scaled boundary finite - element method, International journal for numerical and analytical methods in Geomechanics, 39 (2015) 1-22.

[6] L. Tao, H. Song, S. Chakrabarti, Scaled boundary FEM solution of short-crested wave diffraction by a vertical cylinder, Computer methods in applied mechanics and engineering, 197 (2007) 232-242.

[7] Y. He, H. Yang, A.J. Deeks, An element-free Galerkin scaled boundary method for steady-state heat transfer problems, Numerical heat transfer, Part B: Fundamentals, 64 (2013) 199-217.

[8] S. Natarajan, J.C. Wang, C.M. Song, C. Birk, Isogeometric analysis enhanced by the scaled boundary finite element method, Computer methods in applied mechanics and engineering, 283 (2015) 733-762.

[9] C.M. Song, J.P. Wolf, Semi-analytical representation of stress singularities as occurring in cracks in anisotropic multi-materials with the scaled boundary finite-element method, Computers \& structures, 80 (2002) 183-197.

[10] Z. Yang, Fully automatic modelling of mixed-mode crack propagation using scaled boundary finite element method, Engineering fracture mechanics, 73 (2006) 1711-1731.

[11] E. Ooi, Z. Yang, A hybrid finite element-scaled boundary finite element method for crack propagation modelling, Computer methods in applied mechanics and engineering, 199 (2010) 1178-1192.

[12] E.T. Ooi, Z.J. Yang, Modelling dynamic crack propagation using the scaled boundary finite element method, International journal for numerical methods in engineering, 88 (2011) 329-349.

[13] S. Dai, C. Augarde, C. Du, D. Chen, A fully automatic polygon scaled boundary finite element method for modelling crack propagation, Engineering fracture mechanics, 133 (2015) 163-178.

[14] E.T. Ooi, C.M. Song, F. Tin-Loi, A scaled boundary polygon formulation for elasto-plastic analyses, Computer methods in applied mechanics and engineering, 268 (2014) 905-937.

[15] M.S. Chowdhury, C.M. Song, W. Gao, Probabilistic fracture mechanics by using Monte Carlo simulation and the scaled boundary finite element method, Engineering fracture mechanics, 78 (2011) 2369-2389.

[16] M.S. Chowdhury, C. Song, W. Gao, Probabilistic fracture mechanics with uncertainty in crack size and orientation using the scaled boundary finite element method, Computers \& structures, 137 (2014) 93-103. 
[17] C. Jiang, X. Long, X. Han, Y. Tao, J. Liu, Probability-interval hybrid reliability analysis for cracked structures existing epistemic uncertainty, Engineering fracture mechanics, 112 (2013) 148-164.

[18] X.Y. Long, C. Jiang, X. Han, W. Gao, Stochastic response analysis of the scaled boundary finite element method and application to probabilistic fracture mechanics, Computers \& structures, 153 (2015) 185-200.

[19] X.Y. Long, C. Jiang, X. Han, W. Gao, D.Q. Zhang, Stochastic fracture analysis of cracked structures with random field property using the scaled boundary finite element method, International journal of fracture, 195 (2015) 1-14.

[20] T.-H. Lee, K.M. Mosalam, Probabilistic fiber element modeling of reinforced concrete structures, Computers \& structures, 82 (2004) 2285-2299.

[21] V.V. Silberschmidt, Crack propagation in random materials: computational analysis, Computational materials science, 26 (2003) 159-166.

[22] W.-F. Wu, H.-C. Cheng, C.-K. Kang, Random field formulation of composite laminates, Composite structures, 49 (2000) 87-93.

[23] R.G. Ghanem, P.D. Spanos, Stochastic finite elements: a spectral approach, Springer, 1991.

[24] R.G. Ghanem, P.D. Spanos, Spectral stochastic finite-element formulation for reliability analysis, Journal of engineering mechanics, 117 (1991) 2351-2372.

[25] M. Kleiber, T.D. Hien, The stochastic finite element method: basic perturbation technique and computer implementation, Wiley, 1992.

[26] M. Papadrakakis, V. Papadopoulos, Robust and efficient methods for stochastic finite element analysis using Monte Carlo simulation, Computer methods in applied mechanics and engineering, 134 (1996) 325-340.

[27] B. Sudret, A. Der Kiureghian, Stochastic finite element methods and reliability: a state-of-the-art report, Department of civil and environmental engineering, university of California, 2000.

[28] V. Papadopoulos, M. Papadrakakis, Finite-element analysis of cylindrical panels with random initial imperfections, Journal of engineering mechanics, 130 (2004) 867-876.

[29] G. Stefanou, The stochastic finite element method: past, present and future, Computer methods in applied mechanics and engineering, 198 (2009) 1031-1051.

[30] C.M. Song, J.P. Wolf, Consistent infinitesimal finite-element cell method: three dimensional vector wave equation, International journal for numerical methods in engineering, 39 (1996) 2 189-2208.

[31] C.M. Song, J.P. Wolf, The scaled boundary finite-element method-alias consistent infinitesimal finite element cell method for elastodynamics, Computer methods in applied mechanics and engineering, 147 (1997) 329-355.

[32] A.J. Deeks, J.P. Wolf, A virtual work derivation of the scaled boundary finite-element method for elastostatics, Computational mechanics, 28 (2002) 489-504.

[33] M. Steven Greene, Y. Liu, W. Chen, W.K. Liu, Computational uncertainty analysis in multiresolution materials via stochastic constitutive theory, Computer methods in applied mechanics and engineering, 200 (2011) 309-325.

[34] R. Ghanem, The nonlinear Gaussian spectrum of log-normal stochastic processes and variables, Journal of applied mechanics, 66 (1999) 964-973.

[35] K.E. Atkinson, The numerical solution of integral equations of the second kind, Cambridge 
university press, 1997.

[36] W. Betz, I. Papaioannou, D. Straub, Numerical methods for the discretization of random fields by means of the Karhunen-Loeve expansion, Computer methods in applied mechanics and engineering, 271 (2014) 109-129.

[37] C. Li, A. Der Kiureghian, Optimal discretization of random fields, Journal of engineering mechanics, 119 (1993) 1136-1154.

[38] E.T. Ooi, C.M. Song, F. Tin-Loi, Z. Yang, Polygon scaled boundary finite elements for crack propagation modelling, International journal for numerical methods in engineering, 91 (2012) 319-342.

[39] X.Y. Long, C. Jiang, X. Han, W. Gao, R.G. Bi, Sensitivity analysis of the scaled boundary finite element method for elastostatics, Computer methods in applied mechanics and engineering, 276 (2014) 212-232.

[40] L. Li, Y. Hu, X. Wang, A parallel way for computing eigenvector sensitivity of asymmetric damped systems with distinct and repeated eigenvalues, Mechanical systems and signal processing, 30 (2012) 61-77.

[41] X.Y. Long, C. Jiang, X. Han, New method for eigenvector-sensitivity analysis with repeated eigenvalues and eigenvalue derivatives, AIAA journal, 53 (2015) 1226-1235.

[42] L. Li, Y. Hu, X. Wang, L. Ling, Eigensensitivity analysis for asymmetric nonviscous systems, AIAA journal, 51 (2012) 738-741.

[43] L. Li, Y. Hu, X. Wang, Design sensitivity and Hessian matrix of generalized eigenproblems, Mechanical systems and signal processing, 43 (2014) 272-294.

[44] G.R. Irwin, Fracture dynamics, Fracturing of metals, 147 (1948) 166.

[45] C.M. Song, Evaluation of power-logarithmic singularities, T-stresses and higher order terms of in-plane singular stress fields at cracks and multi-material corners, Engineering fracture mechanics, 72 (2005) 1498-1530. 


\section{Figure captions}

Fig. 1 The concept of scaled boundary finite element method

Fig. 2 The concept of random field

Fig. 3 Generation of the polygon mesh

Fig. 4 Cracked polygon discretized by SBFEM

Fig. 5 The cantilever beam

Fig. 6 Effects of $M$ on the standard deviation of the displacement in the $y$ direction of node $\mathrm{C}$

Fig. 7 The means and standard deviations of the displacements for the cantilever beam

Fig. 8 The edge cracked plate under tension

Fig. 9 Effects of $M$ on the standard deviation of $K_{\mathrm{I}}(\theta)$

Fig. 10 The means and standard deviations of the displacements for the edge cracked plate

Fig. 11 The inclined edge cracked plate under tension

Fig. 12 Samples of the random fields for the inclined edge cracked plate

Fig. 13 Effects of $M$ on the standard deviations of the SIFs

Fig. 14 The edge cracked plate with a hole under tension

Fig. 15 Integration points of the Nyström method (EOLE)

Fig. 16 Samples of the random fields for the edge cracked plate with a hole

Fig. 17 Effects of $M$ on the standard deviation of $K_{\mathrm{I}}(\theta)$

Fig. 18 The means and standard deviations of the displacements for the edge cracked plate with a hole 
Table captions

Table 1 The statistical moments of the displacements of the polygon corresponding to node $\mathrm{C}$

Table 2 The derivatives of the SIFs with respect to the normal variables $\varsigma_{i}$

Table 3 The results of the SIFs under random field property for the horizontal edge cracked plate

Table 4 The derivatives of the SIFs with respect to the normal variables $\varsigma_{i}$

Table 5 The results of the SIFs under random field property for the inclined edge cracked plate

Table 6 Eigensolutions of the IEVP

Table 7 The results of $K_{\mathrm{I}}$ under random field property for the edge cracked plate with a hole 



Fig. 1 The concept of scaled boundary finite element method: (a) SBFEM sub-domains and (b) sub-domain 1. 


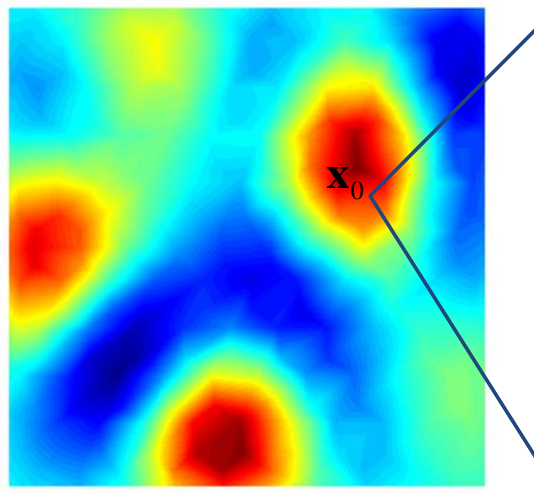

Random field

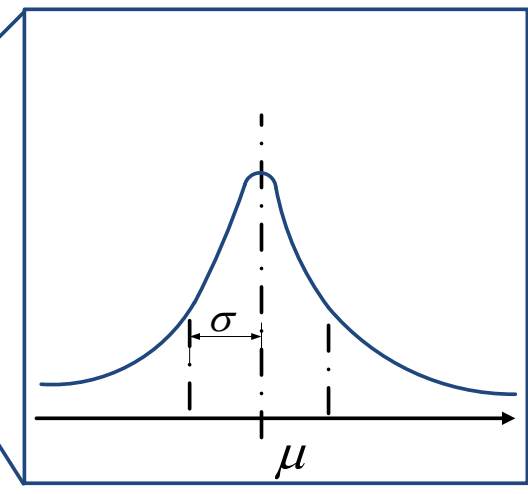

Random variable

Fig. 2 The concept of random field. 

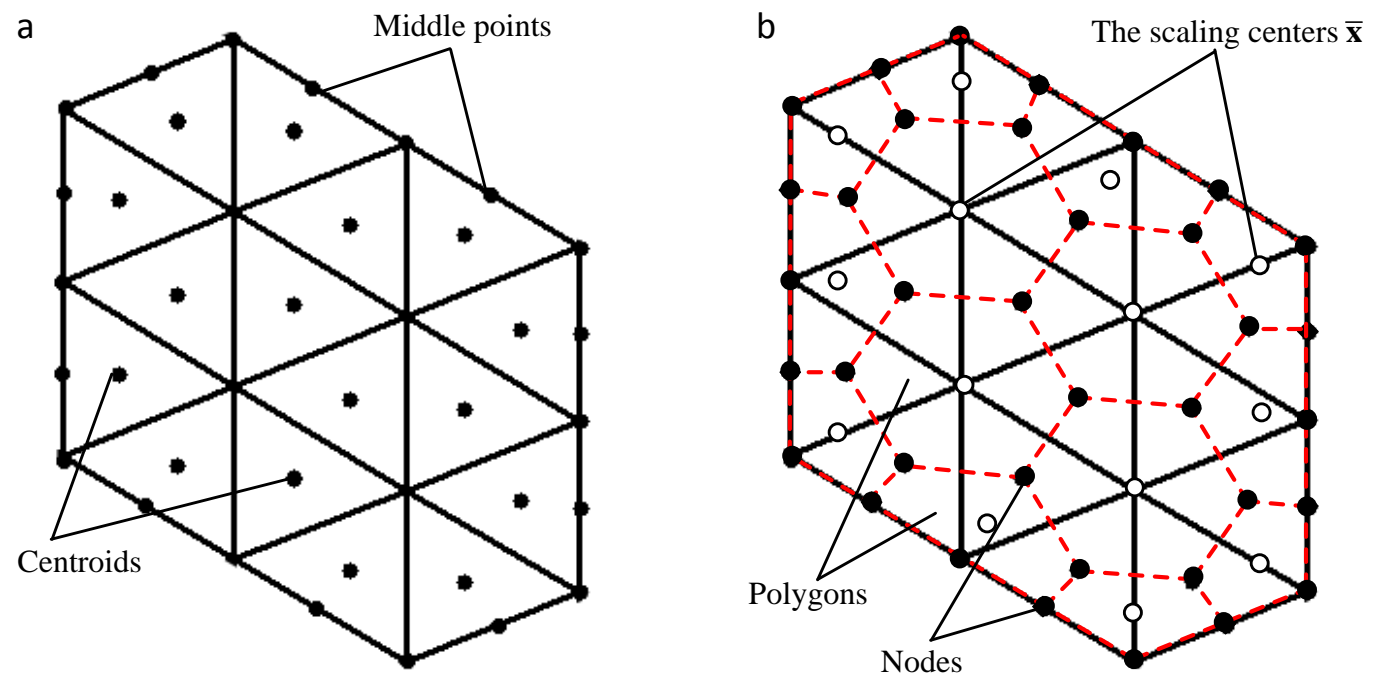

Fig. 3 Generation of the polygon mesh: (a) triangular mesh and (b) polygon mesh. 


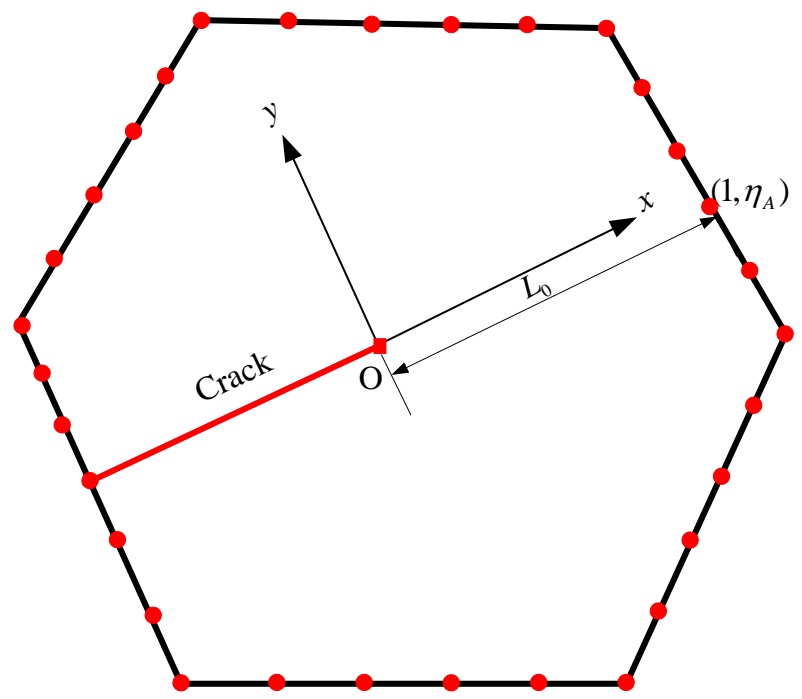

Fig. 4 Cracked polygon discretized by the SBFEM. 
a

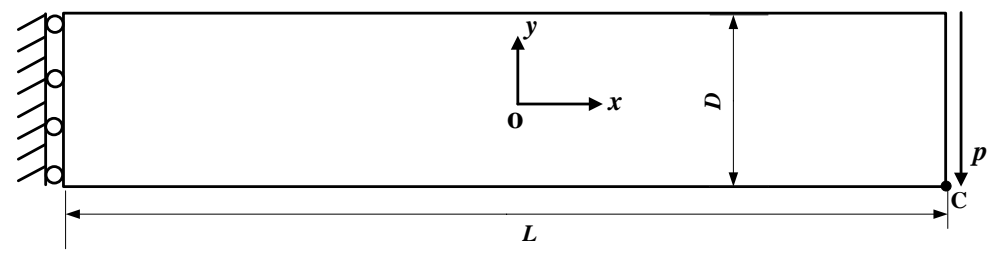

b

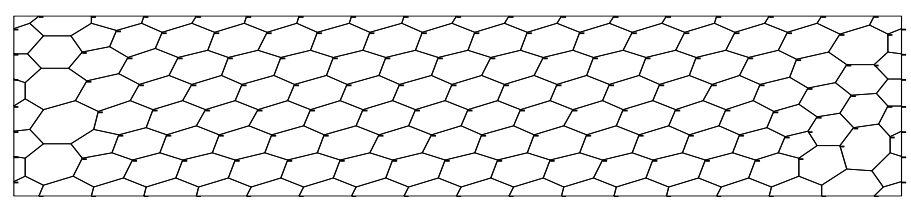

Fig. 5 The cantilever beam: (a) geometry and (b) polygon mesh. 


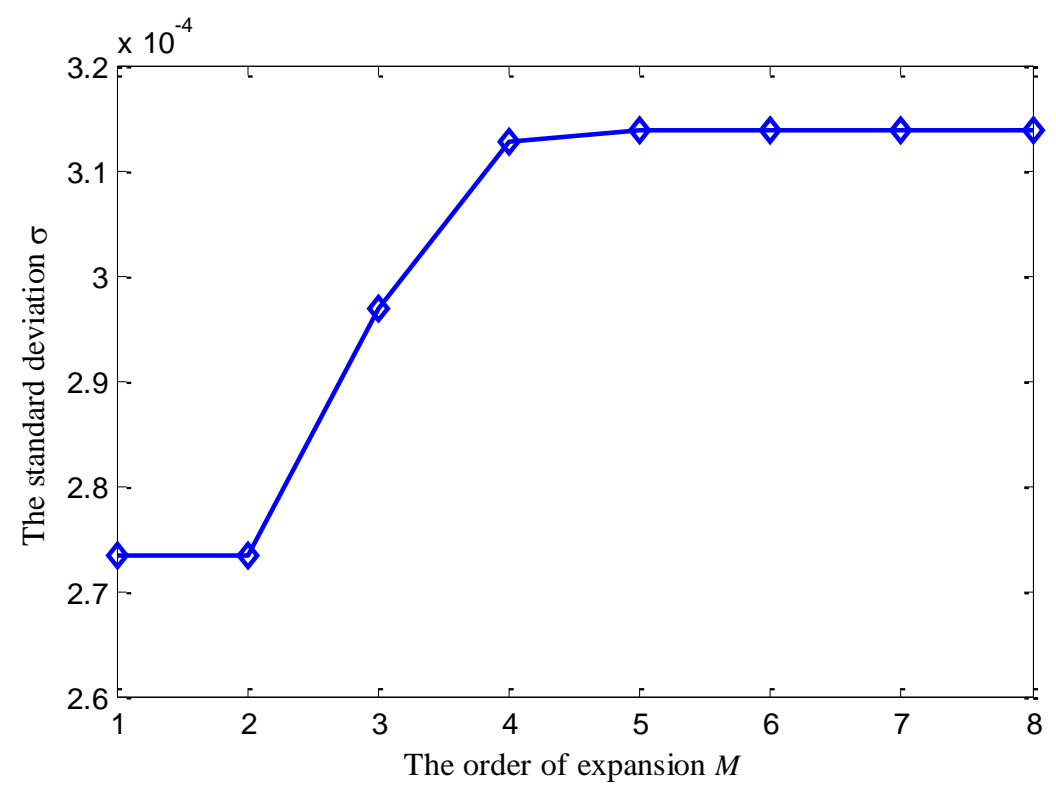

Fig. 6 Effects of $M$ on the standard deviation of displacement in the $y$ direction of node $C$. 
a
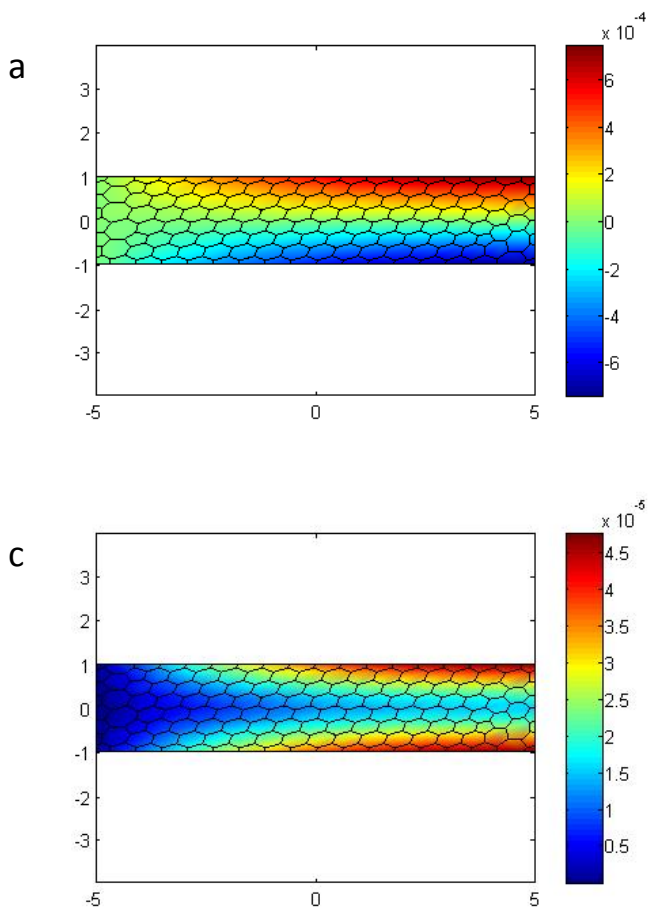

b

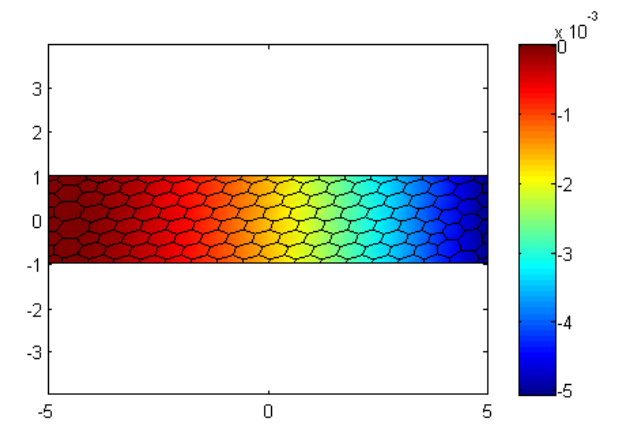

d



Fig. 7 The means and standard deviations of the displacements for the cantilever beam: (a) mean in the $x$ direction, (b) mean in the $y$ direction, (c) standard deviation in the $x$ direction and (d) standard deviation in the $y$ direction. 




b

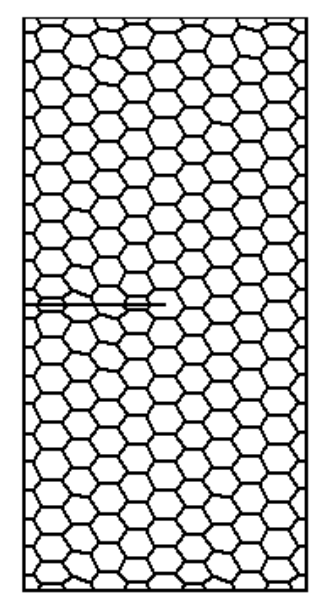

Fig. 8 The edge cracked plate under tension: (a) geometry and (b) polygon mesh. 


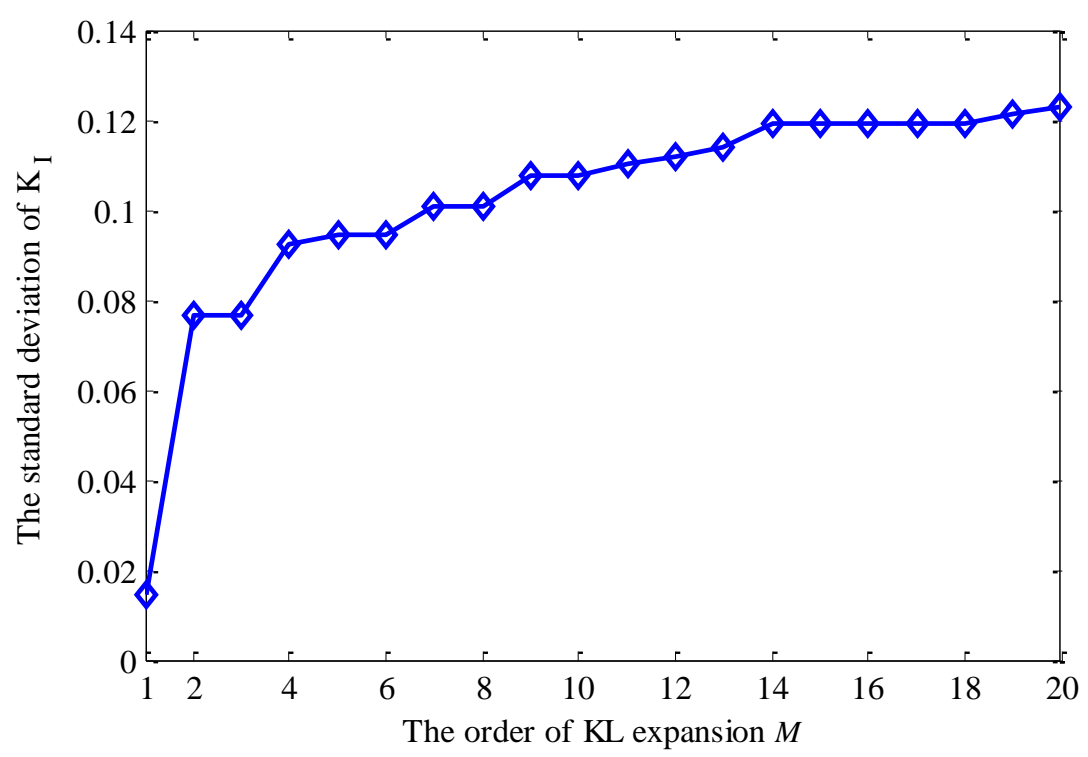

Fig. 9 Effects of $M$ on the standard deviation of $K_{\mathrm{I}}(\theta)$. 
a

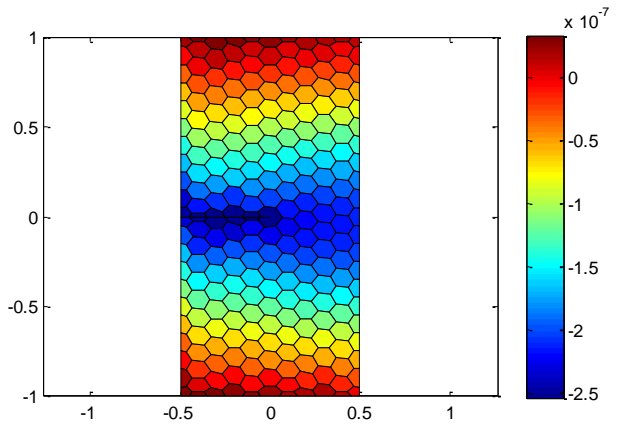

C

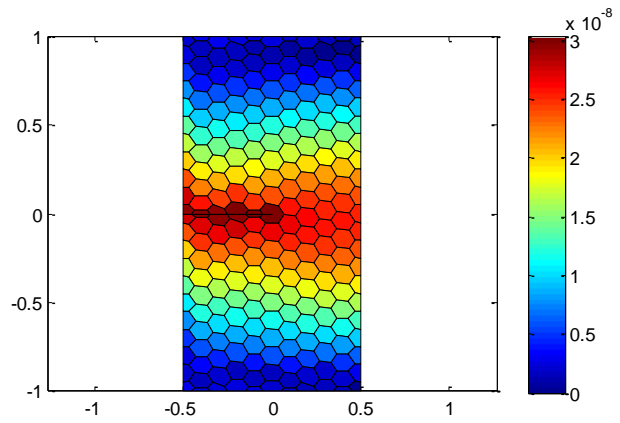

b

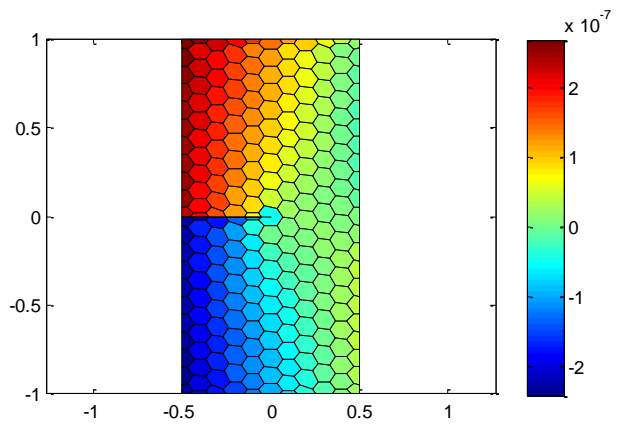

d

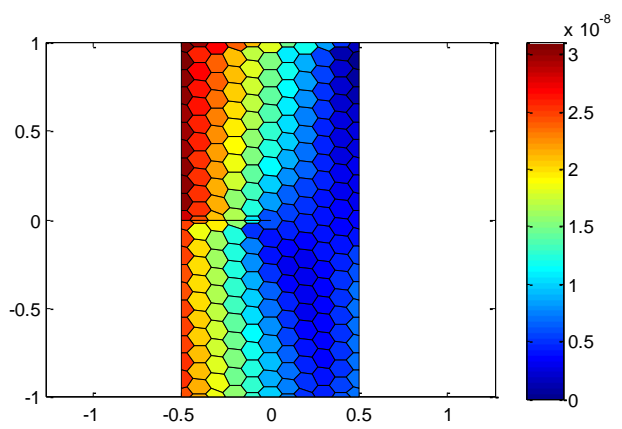

Fig. 10 The means and standard deviations of the displacements for the edge cracked plate: (a) mean in the $x$ direction, (b) mean in the $y$ direction, (c) standard deviation in the $x$ direction and (d) standard deviation in the $y$ direction. 


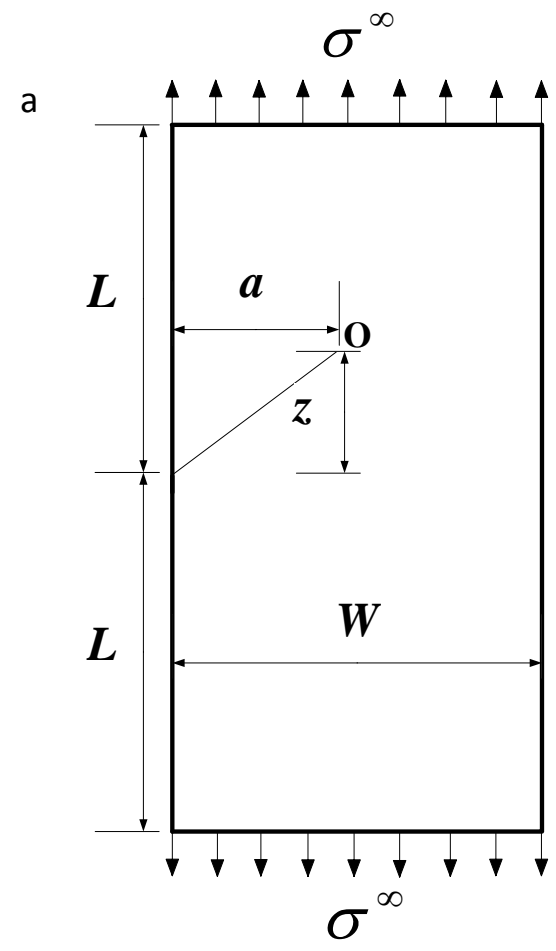

b

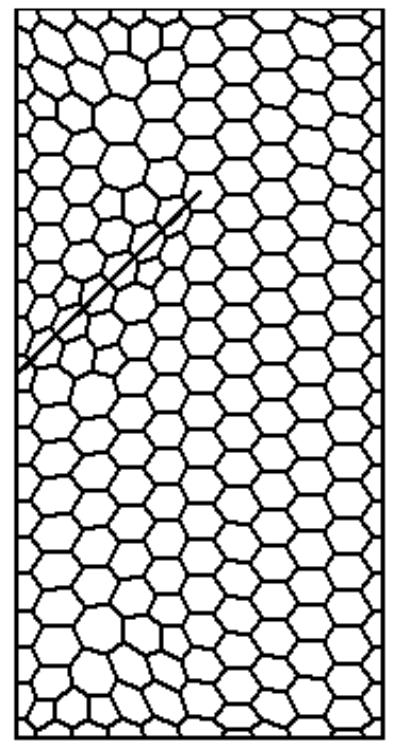

Fig. 11 The inclined edge cracked plate under tension: (a) geometry and (b) polygon mesh. 
a

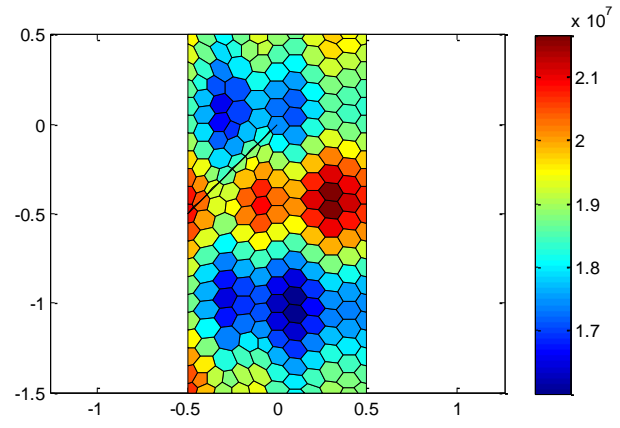

b

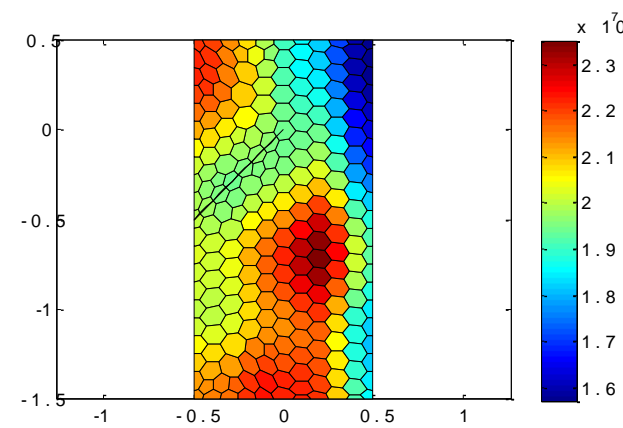

C

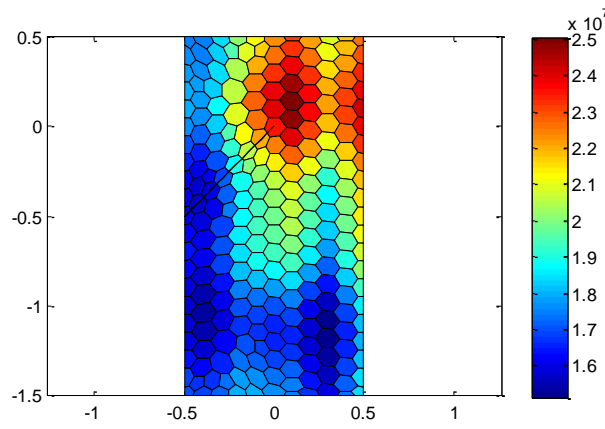

Fig. 12 Samples of the random fields for the inclined edge cracked plate: (a) random field 1, (b) random field 2 and (c) random field 3 
a

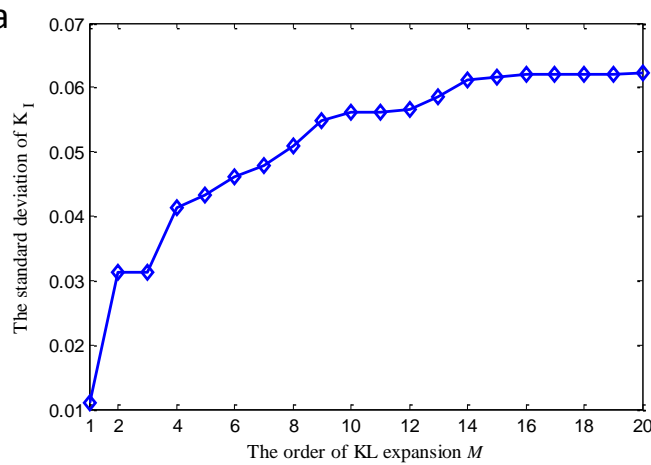

b



Fig. 13 Effects of $M$ on the standard deviations of the SIFs: (a) $K_{\mathrm{I}}(\theta)$ vs. $M$ and (b) $K_{\mathrm{II}}(\theta)$ vs. $M$. 


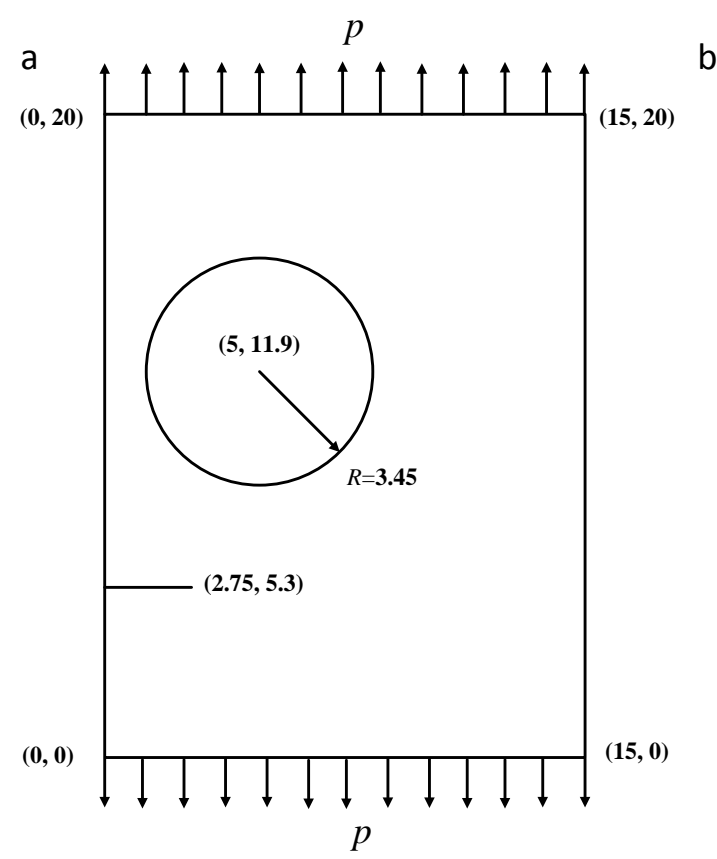

b

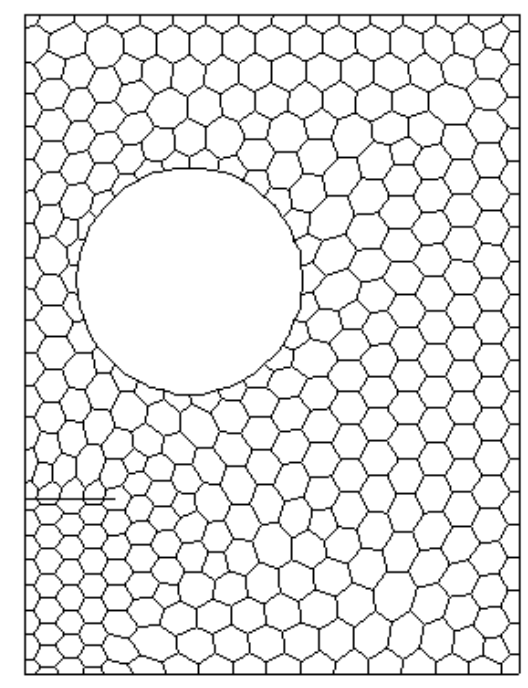

Fig 14 The edge cracked plate with a hole under tension: (a) geometry and (b) polygon mesh. 
a

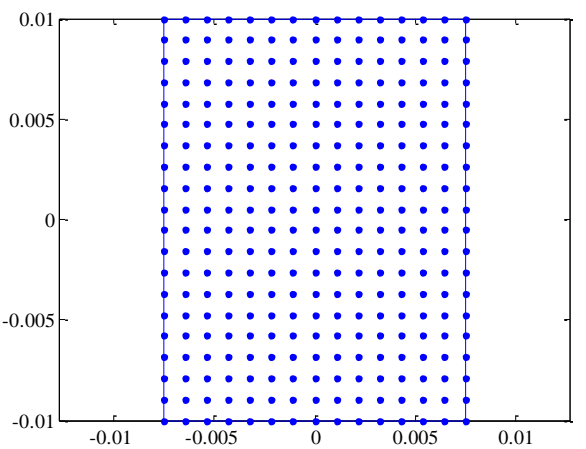

b

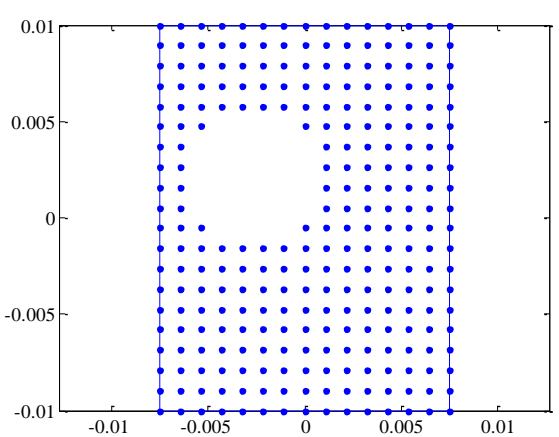

Fig. 15 Integration points of the Nyström method (EOLE): (a) the primitive domain of simple rectangular geometry and (b) the physical domain of non-rectangular geometry. 
a

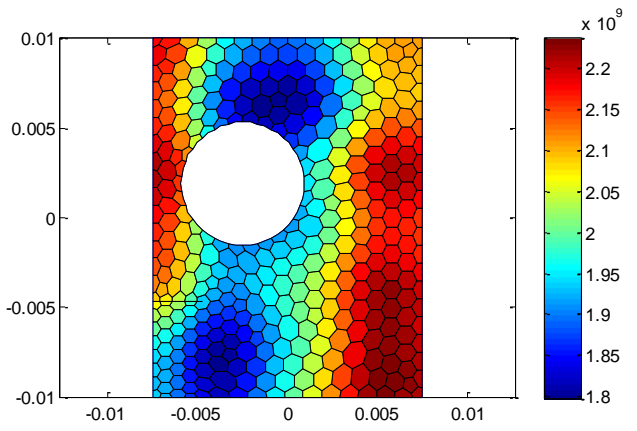

b



C



Fig. 16 Samples of the random fields for the edge cracked plate with a hole: (a) random field 1 , (b) random field 2 and (c) random field 3. 


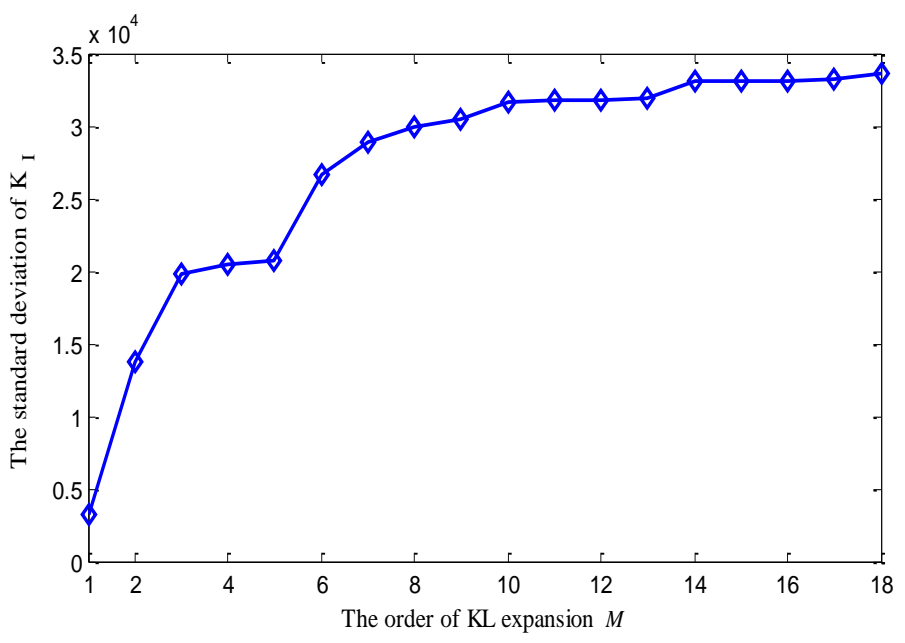

Fig. 17 Effects of $M$ on the standard deviation of $K_{\mathrm{I}}(\theta)$ 
a

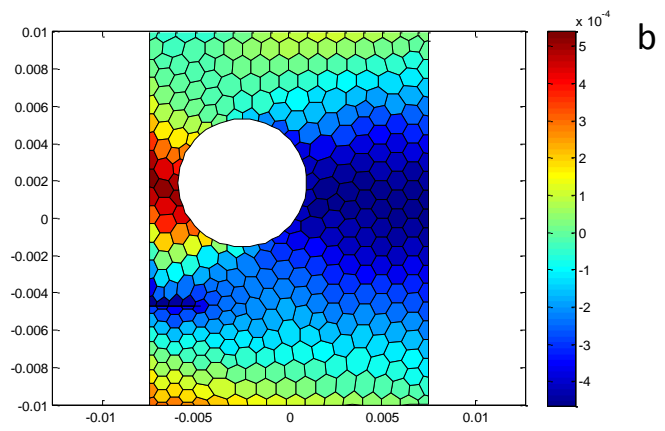

C

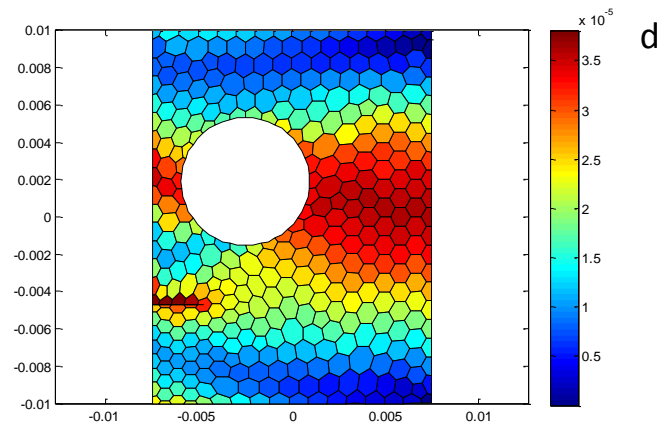

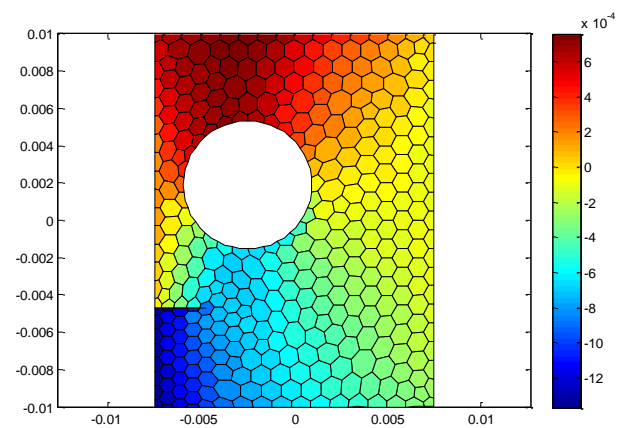

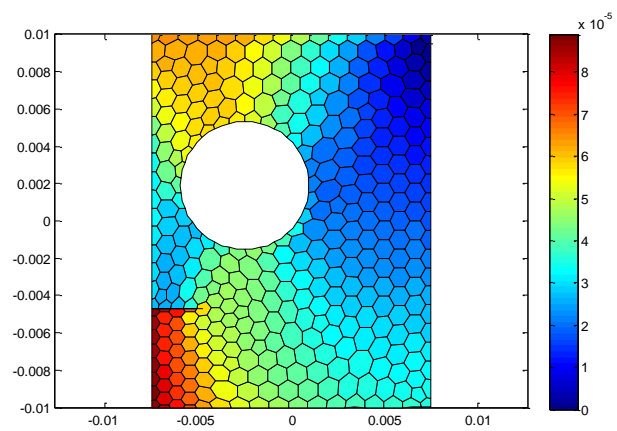

Fig. 18 The means and standard deviations of the displacements for the edge cracked plate with a hole: (a) mean in the $x$ direction, (b) mean in the $y$ direction, (c) standard deviation in the $x$ direction and (d) standard deviation in the $y$ direction. 
Table 1 The statistical moments of the displacements of the polygon corresponding to node C

\begin{tabular}{ccccccc}
\hline DOF & \multicolumn{3}{c}{ Mean $\mu$} & \multicolumn{3}{c}{ Std $\sigma$} \\
\cline { 2 - 7 } & SSBFEM & MCS & $\begin{array}{c}\text { Relative } \\
\text { Error }\end{array}$ & SSBFEM & MCS & $\begin{array}{c}\text { Relative } \\
\text { Error }\end{array}$ \\
\hline 1 & $-6.3449 \mathrm{e}-4$ & $-6.3892 \mathrm{e}-4$ & $0.69 \%$ & $4.1379 \mathrm{e}-5$ & $4.2028 \mathrm{e}-5$ & $1.54 \%$ \\
2 & $-5.0703 \mathrm{e}-3$ & $-5.1051 \mathrm{e}-3$ & $0.68 \%$ & $3.1370 \mathrm{e}-4$ & $3.1926 \mathrm{e}-4$ & $1.74 \%$ \\
3 & $-6.6989 \mathrm{e}-4$ & $-6.7457 \mathrm{e}-4$ & $0.69 \%$ & $4.3369 \mathrm{e}-5$ & $4.4050 \mathrm{e}-5$ & $1.55 \%$ \\
4 & $-4.9212 \mathrm{e}-3$ & $-4.9548 \mathrm{e}-3$ & $0.68 \%$ & $3.0486 \mathrm{e}-4$ & $3.1027 \mathrm{e}-4$ & $1.74 \%$ \\
5 & $-7.4152 \mathrm{e}-4$ & $-7.46703 \mathrm{e}-4$ & $0.69 \%$ & $4.7424 \mathrm{e}-5$ & $4.8172 \mathrm{e}-5$ & $1.55 \%$ \\
6 & $-4.8469 \mathrm{e}-3$ & $-4.8801 \mathrm{e}-3$ & $0.68 \%$ & $3.0046 \mathrm{e}-4$ & $3.0579 \mathrm{e}-4$ & $1.74 \%$ \\
7 & $-7.4269 \mathrm{e}-4$ & $-7.4787 \mathrm{e}-4$ & $0.69 \%$ & $4.7475 \mathrm{e}-5$ & $4.8224 \mathrm{e}-5$ & $1.55 \%$ \\
8 & $-5.0706 \mathrm{e}-3$ & $-5.1054 \mathrm{e}-3$ & $0.68 \%$ & $3.1371 \mathrm{e}-4$ & $3.1927 \mathrm{e}-4$ & $1.74 \%$ \\
\hline
\end{tabular}


Table 2 The derivatives of the SIFs with respect to the normal variables $\varsigma_{i}$

\begin{tabular}{|c|c|c|c|c|c|c|}
\hline & The derivatives of SIFs & $\varsigma_{1}$ & $\varsigma_{2}$ & $\varsigma_{3}$ & $\varsigma_{4}$ & $\varsigma_{5}$ \\
\hline \multirow{3}{*}{$\partial K_{\mathrm{I}}(\theta) / \partial \varsigma_{i}$} & $\begin{array}{l}\text { Proposed sensitivity analysis } \\
\text { method }\end{array}$ & 0.0097 & -0.0503 & -0.0005 & 0.0346 & 0.0128 \\
\hline & Central difference method & 0.0096 & -0.0503 & -0.0005 & 0.0346 & 0.0128 \\
\hline & Relative error & $1.03 \%$ & $0 \%$ & $0 \%$ & $0 \%$ & $0 \%$ \\
\hline \multirow{3}{*}{$\partial K_{\mathrm{II}}(\theta) / \partial \varsigma_{i}$} & $\begin{array}{l}\text { Proposed sensitivity analysis } \\
\text { method }\end{array}$ & 0.0001 & -0.0005 & 0.0146 & 0.0003 & 0.0001 \\
\hline & Central difference method & 0.0001 & -0.0005 & 0.0145 & 0.0003 & 0.0001 \\
\hline & Relative error & $0 \%$ & $0 \%$ & $0.68 \%$ & $0 \%$ & $0 \%$ \\
\hline
\end{tabular}


Table 3 The results of the SIFs under random field property for the horizontal edge cracked plate

\begin{tabular}{cccccccc}
\hline \multirow{2}{*}{ SIFs } & \multicolumn{2}{c}{ Mean $\mu$} & \multicolumn{3}{c}{ Std $\sigma$} \\
\cline { 2 - 7 } & SSBFEM & MCS & $\begin{array}{c}\text { Relative } \\
\text { error }\end{array}$ & SSBFEM & MCS & $\begin{array}{c}\text { Relative } \\
\text { error }\end{array}$ \\
\hline$K_{\mathrm{I}}$ & 3.4829 & 3.4836 & $0.02 \%$ & 0.1192 & 0.1195 & $0.25 \%$ \\
$K_{\mathrm{II}}$ & 0.0385 & 0.0381 & $1.05 \%$ & 0.0275 & 0.0274 & $0.36 \%$ \\
\hline
\end{tabular}


Table 4 The derivatives of the SIFs with respect to the normal variables $\varsigma_{i}$

\begin{tabular}{llccccc}
\hline \multicolumn{1}{c}{ The derivatives of SIFs } & $\varsigma_{1}$ & $\varsigma_{2}$ & $\varsigma_{3}$ & $\varsigma_{4}$ & $\varsigma_{5}$ \\
\hline \multirow{2}{*}{$\partial K_{\mathrm{I}}(\theta) / \partial \varsigma_{i}$} & Proposed sensitivity analysis & 0.0111 & -0.0293 & -0.0014 & 0.0269 & 0.0130 \\
& method & 0.0111 & -0.0292 & -0.0014 & 0.0270 & 0.0131 \\
& Central difference method & $0 \%$ & $0.34 \%$ & $0 \%$ & $0.37 \%$ & $0.77 \%$ \\
& Relative error & & & & & \\
& Proposed sensitivity analysis & 0.0006 & -0.0254 & 0.0057 & 0.0104 & -0.0070 \\
$\partial K_{\mathrm{II}}(\theta) / \partial \varsigma_{i}$ & method & 0.0006 & -0.0254 & 0.0056 & 0.0105 & -0.0070 \\
& Central difference method & $0.00 \%$ & $0.96 \%$ & $0 \%$ \\
\hline
\end{tabular}


Table 5 The results of the SIFs under random field property for the inclined edge cracked plate

\begin{tabular}{ccccccc}
\hline \multirow{2}{*}{ SIFs } & \multicolumn{2}{c}{ Mean $\mu$} & \multicolumn{3}{c}{ Std $\sigma$} \\
\cline { 2 - 7 } & SSBFEM & MCS & $\begin{array}{c}\text { Relative } \\
\text { error }\end{array}$ & SSBFEM & MCS $\begin{array}{c}\text { Relative } \\
\text { error }\end{array}$ \\
\hline$K_{\text {I }}$ & 2.9941 & 2.9980 & $0.13 \%$ & 0.0620 & 0.0622 & $0.32 \%$ \\
$K_{\text {II }}$ & 1.0546 & 1.0530 & $0.15 \%$ & 0.0382 & 0.0380 & $0.53 \%$ \\
\hline
\end{tabular}


Table 6 Eigensolutions of the IEVP

\begin{tabular}{cccc}
\hline$\gamma_{j}$ & Nyström method(EOLE) & Analytical method & Relative error \\
\hline 1 & $1.0669 \mathrm{e}-04$ & $1.1170 \mathrm{e}-04$ & $4.49 \%$ \\
2 & $3.7944 \mathrm{e}-05$ & $3.7997 \mathrm{e}-05$ & $0.14 \%$ \\
3 & $3.0485 \mathrm{e}-05$ & $2.9895 \mathrm{e}-05$ & $1.97 \%$ \\
4 & $1.5673 \mathrm{e}-05$ & $1.5264 \mathrm{e}-05$ & $2.68 \%$ \\
5 & $1.1484 \mathrm{e}-05$ & $1.0902 \mathrm{e}-05$ & $5.34 \%$ \\
\hline
\end{tabular}


Table 7 The results of $K_{\mathrm{I}}$ under random field property for the edge cracked plate with a hole

\begin{tabular}{ccccccc}
\hline \multirow{2}{*}{ SIFs } & \multicolumn{3}{c}{ Mean $\mu$} & \multicolumn{3}{c}{ Std $\sigma$} \\
\cline { 2 - 7 } & SSBFEM & MCS & $\begin{array}{c}\text { Relative } \\
\text { error }\end{array}$ & SSBFEM & MCS & $\begin{array}{c}\text { Relative } \\
\text { error }\end{array}$ \\
\hline$K_{\mathrm{I}}$ & $1.2993 \mathrm{e} 6$ & $1.2979 \mathrm{e} 6$ & $0.11 \%$ & $3.3106 \mathrm{e} 4$ & $3.2855 \mathrm{e} 4$ & $0.76 \%$ \\
\hline
\end{tabular}

